TRANSACTIONS OF THE

AMERICAN MATHEMATICAL SOCIETY

Volume 360, Number 10, October 2008, Pages 5067-5087

S 0002-9947(08)04393-6

Article electronically published on May 19, 2008

\title{
ON $p$-PERMUTATION EQUIVALENCES: BETWEEN RICKARD EQUIVALENCES AND ISOTYPIES
}

\author{
ROBERT BOLTJE AND BANGTENG XU
}

\begin{abstract}
Broué and Rickard defined in their landmark papers from 1990 and 1996 the notions of an isotypy and a splendid equivalence between $p$-blocks of finite groups. Here, we define a notion of equivalence, which we call a $p$ permutation equivalence, that implies an isotypy and is implied by a splendid equivalence. Moreover, we study properties of $p$-permutation equivalences.
\end{abstract}

\section{INTRODUCTION}

Let $G$ and $H$ be finite groups and let $A$ and $B$ be $p$-blocks of $G$ and $H$, respectively, which have a common subgroup $D$ of $G$ and $H$ as a defect group. Broué defined in Br90 the notions of a perfect isometry and of an isotypy between $A$ and $B$. Roughly speaking, a perfect isometry between $A$ and $B$ is an isometry between the character groups of $A$ and $B$ satisfying a certain arithmetic property with respect to $p$. An isotypy between $A$ and $B$ is a family of perfect isometries between the centralizers of subgroups $P \leqslant D$ in $G$ and $H$, which are compatible in a sense that involves the generalized decomposition map. In Ri96, Rickard lifted the notion of an isotypy to a categorical level by introducing the notion of a splendid equivalence. This consists of a finite chain complex of $p$-permutation $(A, B)$-bimodules, which via tensoring induces an equivalence between the homotopy categories of $A$ and $B$. In the case of principal blocks, Rickard proved that taking the Brauer construction for the various subgroups $P \leqslant D$ of such a chain complex and then taking its generalized character results in an isotypy. Broué's abelian defect group conjecture states that when $D$ is abelian, $H=N_{G}(D)$, and $A$ and $B$ are Brauer correspondents, then there exists an isotypy, or even stronger, a splendid equivalence between $A$ and $B$.

Rickard's work indicates that, if one wants to work on a Grothendieck group level instead of a categorical level, the appropriate home should be the trivial source ring of $G \times H$ rather than the family of character rings of all the centralizers of subgroups $P \leqslant D$ in $G$ and $H$. Consequently, we define a $p$-permutation equivalence between $A$ and $B$ as an element $\gamma$ in the representation ring of $p$-permutation $(A, B)$ bimodules which satisfies $\gamma \cdot \gamma^{\llcorner}=[A]$ and $\gamma^{\llcorner} \cdot \gamma=[B]$ in the representation rings of $(A, A)$-bimodules and $(B, B)$-bimodules, respectively. Here, $\gamma^{\sim}$ denotes the dual

Received by the editors October 5, 2005.

2000 Mathematics Subject Classification. Primary 20C20, 20C15, 19A22.

The first author's research was supported by the NSF, DMS-0200592.

(C)2008 American Mathematical Society

Reverts to public domain 28 years from publication 
of $\gamma$. We understand the present paper as a first systematic study of the notion of a $p$-permutation equivalence. It is not difficult to see that a splendid equivalence induces a $p$-permutation equivalence, by just taking the alternating sum of the classes of the terms of the chain complex. In our main theorem, Theorem 1.11, we establish several properties of $p$-permutation equivalences under the assumption that $D$ is abelian and that the Frobenius categories of $A$ and $B$ are equivalent. We show that $p$-permutation equivalences induce isotypies, which is not surprising but harder to show than in the case of a splendid equivalence. Moreover, we show that, by applying the Brauer construction with respect to a subgroup $P \leqslant D$, one obtains a $p$-permutation equivalence between associated blocks of the centralizers of $P$ in $G$ and $H$. Moreover, it follows that the Brauer categories are equivalent. Additional technical difficulties compared to the case of splendid equivalences occur, since one no longer has objects, as for instance the local endomorphism ring of the splendid chain complex as tools.

Along the way of the proof of the main theorem we prove some results that might be of independent interest. In Theorem 2.4 (resp. Corollary 2.6) we establish commutative diagrams that interpret the generalized Brauer construction on $p$-monomial modules (resp. the Brauer construction on $p$-permutation modules) as lifts of the generalized decomposition map. Brauer's Second Main Theorem is then an immediate consequence. It is necessary to work with $p$-monomial modules, since (in contrast to $p$-permutation modules) their representation ring maps surjectively onto the character ring. In Lemma 3.5 and Corollary 3.6 we prove that (under suitable assumptions) a certain natural map between the generalized Brauer construction of the tensor product and the tensor product of the generalized Brauer constructions is an isomorphism, provided that one of the factors is a $p$-monomial module.

A $p$-permutation equivalence, as an element in the trivial source ring of $G \times H$, is determined by the Brauer characters of its Brauer constructions with respect to certain diagonal $p$-subgroups $\Delta P$. Since the normalizer of $\Delta P$ is not much bigger than $C_{G}(P) \times C_{H}(P)$, it seems that the notion of a $p$-permutation equivalence is not too far from the notion of an isotypy. We hope that in the future we can lift proven cases of isotypies to $p$-permutation equivalences. Also, we hope that with the help of computer programs we will be able to find (or disprove the existence of) $p$-permutation equivalences for particular groups of interest.

The paper is arranged as follows. In Section 1 we introduce the necessary notation and state the main results. In Section 2 we establish a link between the Brauer construction on $p$-monomial and $p$-permutation modules and the generalized decomposition map. In Section 3 we prove the main results along with some necessary lemmas.

\section{Notation And Statement of MAin RESUlts}

1.1. Notation. Throughout this paper we fix two finite groups $G$ and $H$, a prime $p$, and a $p$-modular system $(K, \mathcal{O}, k)$ with the following properties: $\mathcal{O}$ is a complete discrete valuation ring with maximal ideal $\mathfrak{p}$; its residue field, $k:=\mathcal{O} / \mathfrak{p}$, is algebraically closed of characteristic $p$, and its field of fractions, $K$, has characteristic 0 and contains a root of unity whose order is a multiple of both $\exp (G)$ and $\exp (H)$, the exponents of $G$ and $H$. The canonical surjections $\mathcal{O} \rightarrow k$ and $\mathcal{O} G \rightarrow k G$ will be denoted by $a \mapsto \bar{a}$. To simplify the notation, we often view $\mathcal{O} G, k G$, and $K G$ 
as $(\mathcal{O} G, \mathcal{O} G)$-bimodules in the natural way so that expressions like $\sigma a \tau$ are defined for $\sigma, \tau \in \mathcal{O} G$ and $a$ an element of $\mathcal{O} G, K G$, or $k G$. Similarly, we also view a $k G$ module or $K G$-module $M$ as an $\mathcal{O} G$-module and use the notation $\sigma m$ for $\sigma \in \mathcal{O} G$ and $m \in M$. This is why we can omit the bars in many instances, as for example in Subsection 1.8.

Throughout, we fix primitive idempotents $e$ and $f$ of $Z(\mathcal{O} G)$ and $Z(\mathcal{O} H)$, respectively. We assume that the block algebras $\mathcal{O} G e$ and $\mathcal{O} H f$ have a common defect group $D$ (which is contained in $G$ and in $H$ ).

Whenever $R$ is a commutative ring and $M$ is an $R[G \times H]$-module we also consider $M$ as an $(R G, R H)$-bimodule via

$$
g m h=\left(g, h^{-1}\right) m, \quad m \in M, g \in G, h \in H,
$$

and vice-versa.

By $G_{p^{\prime}}$ we denote the set of elements of $G$ whose order is not divisible by $p$. The notation $U \leqslant G$ (resp. $U<G$ ) indicates that $U$ is a subgroup (resp. proper subgroup) of $G$. For $g \in G$ we denote by $c_{g}: G \rightarrow G$ the conjugation map defined by $c_{g}(x)=g x g^{-1}$ for $x \in G$. Moreover, if $U$ is a subset of $G$ we also write ${ }^{g} U$ instead of $g U g^{-1}$.

1.2. By $T(\mathcal{O} G)$ we denote the representation ring of $p$-permutation $\mathcal{O} G$-modules (or trivial source $\mathcal{O} G$-modules), i.e., direct summands of permutation $\mathcal{O} G$-modules. The isomorphism classes $[M]$ of indecomposable $p$-permutation $\mathcal{O} G$-modules $M$ form a $\mathbb{Z}$-basis of $T(\mathcal{O} G)$, and for any $p$-permutation $\mathcal{O} G$-module $M$ we denote by $[M]$ its associated element in $T(\mathcal{O} G)$, i.e., if $M=M_{1} \oplus \cdots \oplus M_{r}$ is a decomposition into indecomposable submodules, then $[M]:=\left[M_{1}\right]+\cdots+\left[M_{r}\right]$. For a $p$-subgroup $P$ of $G$ and an idempotent $\sigma$ of $Z(\mathcal{O} G)$ we denote by $T^{P}(\mathcal{O} G \sigma)$ the subgroup of $T(\mathcal{O} G)$ generated by the elements $[M]$, where $M$ is a relatively $P$-projective p-permutation $\mathcal{O} G$-module satisfying $\sigma M=M$. Similarly, we define $T^{P}(\mathcal{O} G)$, $T(\mathcal{O} G \sigma)$, and $T(k G), T^{P}(k G \sigma)$, etc. It is well-known that the map induced by the functor $k \otimes_{\mathcal{O}}$ - induces an isomorphism $\nu_{G}: T^{P}(\mathcal{O} G \sigma) \rightarrow T^{P}(k G \sigma)$.

By a $p$-permutation $(\mathcal{O} G, \mathcal{O} H)$-bimodule we will always mean a $p$-permutation $\mathcal{O}[G \times H]$-module viewed as a bimodule in the above way. For a $p$-subgroup $S$ of $G \times$ $H$ and idempotents $\sigma$ (resp. $\tau$ ) of $Z(\mathcal{O} G)$ (resp. $Z(\mathcal{O} H)$ ) we set $T^{S}(\mathcal{O} G \sigma, \mathcal{O} H \tau):=$ $T^{S}\left(\mathcal{O}[G \times H]\left(\sigma \otimes \tau^{*}\right)\right)$ by identifying $\mathcal{O}[G \times H]$ with $\mathcal{O} G \otimes_{\mathcal{O}} \mathcal{O} H$ in the obvious way, and we similarly do so for $k$ instead of $\mathcal{O}$. Here, $-{ }^{*}: \mathcal{O} H \rightarrow \mathcal{O} H$ denotes the $\mathcal{O}$-algebra anti-isomorphism given by $h \mapsto h^{-1}$ for $h \in H$. For any subgroup $P$ of $D$ we set $\Delta P:=\{(x, x) \mid x \in P\}$ and we view $\Delta P$ as a subgroup of $G \times H$. Note that $-\otimes_{\mathcal{O} H}-$ induces a $\mathbb{Z}$-bilinear map

$$
T(\mathcal{O} G, \mathcal{O} H) \times T(\mathcal{O} H, \mathcal{O} L) \rightarrow T(\mathcal{O} G, \mathcal{O} L), \quad(\alpha, \beta) \mapsto \alpha_{H} \beta,
$$

for any third group $L$.

For $\sigma$ and $\tau$ as above, taking the $\mathcal{O}$-dual $M^{\sim}:=\operatorname{Hom}_{\mathcal{O}}(M, \mathcal{O})$ of a $p$-permutation $(\mathcal{O} G \sigma, \mathcal{O} H \tau)$-bimodule $M$ results in a $p$-permutation $(\mathcal{O} H \tau, \mathcal{O} G \sigma)$-bimodule and induces an isomorphism

$$
T(\mathcal{O} G \sigma, \mathcal{O} H \tau) \rightarrow T(\mathcal{O} H \tau, \mathcal{O} G \sigma), \quad \alpha \mapsto \alpha^{\check{2}} .
$$

We will use the same notations $-_{H}-$ and $-{ }^{`}$ from $(1.2 . \mathrm{a})$ and $(1.2 . \mathrm{b})$ for the maps with $\mathcal{O}$ replaced by $k$. 
The following definition is modeled after Rickard's notion of a splendid equivalence.

1.3. Definition. A p-permutation equivalence between $\mathcal{O} G e$ and $\mathcal{O} H f$ is an element $\gamma \in T^{\Delta D}(\mathcal{O} G e, \mathcal{O} H f)$ satisfying

$$
\gamma_{H} \gamma^{\check{L}}=[\mathcal{O} G e] \in T(\mathcal{O} G e, \mathcal{O} G e)
$$

and

$$
\gamma_{\dot{G}}^{\sim} \gamma=[\mathcal{O H f}] \in T(\mathcal{O} H f, \mathcal{O} H f) .
$$

1.4. Recall from [Ri96] that a splendid equivalence between $\mathcal{O} G e$ and $\mathcal{O H f}$ is a finite chain complex $X_{*}$ consisting of $\Delta D$-projective $p$-permutation $(\mathcal{O} G e, \mathcal{O H f})$ bimodules $X_{n}$ such that one has homotopy equivalences

$$
X_{*} \otimes_{\mathcal{O} H}\left(X_{*}\right)^{\sim} \simeq \mathcal{O} G e \text { and }\left(X_{*}\right)^{\sim} \otimes_{\mathcal{O} G} X_{*} \simeq \mathcal{O H f}
$$

of chain complexes of $(\mathcal{O G e}, \mathcal{O} G e)$-bimodules and $(\mathcal{O H f}, \mathcal{O H f})$-bimodules, where $\left(X_{*}\right)^{2}$ denotes the $\mathcal{O}$-dual of $X_{*}$ with terms $\operatorname{Hom}_{\mathcal{O}}\left(X_{n}, \mathcal{O}\right)$, and where the right hand sides of the above homotopy equivalences denote the chain complexes with only one nonzero term of the form $\mathcal{O} G e$ and $\mathcal{O H f}$ located in degree 0.

For an arbitrary finite chain complex $C_{*}$ of $p$-permutation $\mathcal{O} G$-modules we set

$$
\gamma_{C_{*}}:=\sum_{n \in \mathbb{Z}}(-1)^{n}\left[C_{n}\right] \in T(\mathcal{O} G) .
$$

1.5. Theorem. Let $X_{*}$ be a splendid equivalence between $\mathcal{O} G e$ and $\mathcal{O H f}$. Then $\gamma:=\gamma_{X_{*}}$ is a p-permutation equivalence between $\mathcal{O} G e$ and $\mathcal{O} H f$.

Proof. We have $\gamma_{\left(X_{*}\right)^{\sim}}=\gamma^{\sim}$ and

$$
\gamma_{H} \gamma^{\llcorner}=\sum_{m, n \in \mathbb{Z}}(-1)^{m+n}\left[X_{m} \otimes_{\mathcal{O} H} X_{n}^{\sim}\right]=\gamma_{X_{*} \otimes_{\mathcal{O} H}\left(X_{*}\right)^{\sim}},
$$

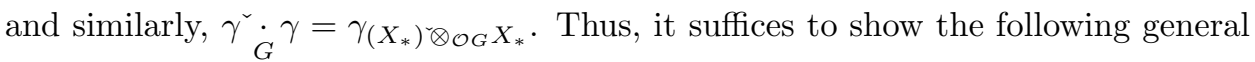
result: Let $C_{*}$ and $D_{*}$ be finite homotopy equivalent chain complexes of $R$-modules for an arbitrary ring $R$. Then

$$
\bigoplus_{n \text { odd }} C_{n-1} \oplus D_{n} \cong \bigoplus_{n \text { even }} C_{n-1} \oplus D_{n}
$$

as $R$-modules. But this follows immediately from considering the mapping cone $E_{*}(f)$ of a homotopy equivalence $f: C_{*} \rightarrow D_{*}$. In fact, $E_{*}(f)$ is contractible and $E_{n}(f)=C_{n-1} \oplus D_{n}$. Moreover, if $E_{*}$ is a contractible chain complex of $R$-modules $E_{n}$, then there exist decompositions $E_{n}=E_{n}^{+} \oplus E_{n}^{-}$into $R$-submodules such that $E_{n}^{+} \cong E_{n+1}^{-}$for all $n \in \mathbb{Z}$.

Under additional assumptions on the local structure of $A$ and $B$, Rickard (in the case of principal blocks; cf. [Ri96]) and Harris (in the general case; cf. Ha99]) proved that applying the Brauer constructions with respect to $\Delta P, P \leqslant D$, to a splendid equivalence results in splendid equivalences on the local levels between certain blocks of the centralizers $C_{G}(P)$ and $C_{H}(P)$. In fact, Harris showed only a slightly weaker version in which no statement is made on the relative projectivity of the chain complexes obtained on the local level. We will obtain the relative projectivity of the local chain complexes in Harris' paper in the case of an abelian defect group as a consequence of Lemma 3.3. We will also show in Theorem 1.11 
that a $p$-permutation equivalence induces $p$-permutation equivalences on the local levels and an isotypy between $\mathcal{O} G e$ and $\mathcal{O} H f$. In order to state the main theorem we will recall the relevant notions for the reader's convenience.

1.6. By $\mathcal{F}_{p}(G)$ we denote the Frobenius category of $G$ with respect to $p$. Its objects are the $p$-subgroups of $G$. For $P, Q \in \mathcal{F}_{p}(G)$ one defines the set $\operatorname{Hom}_{\mathcal{F}_{p}(G)}(Q, P)$ as the set of all group homomorphisms $f \in \operatorname{Hom}(Q, P)$ for which there exists $g \in G$ satisfying $f(x)=c_{g}(x)$ for all $x \in Q$. Moreover, for any subgroup $U \leqslant G$ we define $\mathcal{F}_{p}(U, G)$ as the full subcategory of $\mathcal{F}_{p}(G)$ whose objects are the $p$-subgroups of $U$.

For a $p$-subgroup $P$ of $G$ the Brauer map br $_{P}$ is the $\mathcal{O}$-algebra homomorphism

$$
\operatorname{br}_{P}:(\mathcal{O} G)^{P} \rightarrow Z\left(k\left[C_{G}(P)\right]\right), \quad \sum_{g \in G} \alpha_{g} g \mapsto \sum_{g \in C_{G}(P)} \bar{\alpha}_{g} g,
$$

where $(\mathcal{O} G)^{P}$ denotes the $P$-fixed points of $\mathcal{O} G$ under the conjugation action. It restricts to a map $Z(\mathcal{O} G) \rightarrow Z\left(k\left[N_{G}(P)\right]\right)$. If $\sigma$ is an idempotent in $Z(\mathcal{O} G)$, we can lift the idempotent $\operatorname{br}_{P}(\sigma)$ of $Z\left(k\left[C_{G}(P)\right]\right)$ to an idempotent of $Z\left(\mathcal{O}\left[C_{G}(P)\right]\right)$ which, by abuse of notation, we denote again by $\operatorname{br}_{P}(\sigma)$.

By $\mathcal{B}_{e}(G)$ we denote the Brauer category of $e$. Its objects are the e-subpairs, i.e., pairs $(P, \varepsilon)$ with $P \in \mathcal{F}_{p}(G)$ and $\varepsilon$ a primitive idempotent of $Z\left(\mathcal{O}\left[C_{G}(P)\right]\right)$ with $\operatorname{br}_{P}(e) \varepsilon=\varepsilon$. One can define a partial order $\leqslant$ on the set of $e$-subpairs which is respected by the conjugation action of $G$; cf. AB79] or [Th95]. Using this, for $(P, \varepsilon),(Q, \delta) \in \mathcal{B}_{e}(G)$, one defines $\operatorname{Hom}_{\mathcal{B}_{e}(G)}((Q, \delta),(P, \varepsilon))$ as the set of all homomorphisms $f \in \operatorname{Hom}(Q, P)$ such that there exists $g \in G$ satisfying $f(x)=$ $g x g^{-1}$ for all $x \in Q$ and ${ }^{g}(Q, \delta) \leqslant(P, \varepsilon)$. For any subgroup $U$ of $G$ we denote by $\mathcal{B}_{e}(U, G)$ the full subcategory of $\mathcal{B}_{e}(G)$ whose objects are the $e$-subpairs $(P, \varepsilon)$ with $P \leqslant U$.

1.7. For an $\mathcal{O} G$-module $M$ and a $p$-subgroup $P$ the Brauer construction $\bar{M}(P)$ is defined as

$$
\bar{M}(P):=M^{P} /\left(\sum_{Q<P} \operatorname{tr}_{Q}^{P}\left(M^{Q}\right)+\mathfrak{p} M^{P}\right)
$$

where for $Q \leqslant P$ the relative trace map $\operatorname{tr}_{Q}^{P}: M^{Q} \rightarrow M^{P}$ is defined by $m \mapsto$ $\sum_{x Q \in P / Q} x m$ for $m \in M^{Q}$. Note that $\bar{M}(P)$ is a $k\left[N_{G}(P)\right]$-module. The canonical epimorphism $M^{P} \rightarrow \bar{M}(P)$ will be denoted by $\operatorname{Br}_{P}^{M}$ (or just $\operatorname{Br}_{P}$ ). It is well-known that the Brauer construction induces a homomorphism

$$
T(\mathcal{O} G) \rightarrow T\left(k\left[N_{G}(P)\right]\right), \quad \alpha \mapsto \bar{\alpha}(P)
$$

which composed with the inverse of the isomorphism $\nu_{N_{G}(P)}: T\left(\mathcal{O}\left[N_{G}(P)\right]\right) \rightarrow$ $T\left(k\left[N_{G}(P)\right]\right)$ yields a map

$$
T(\mathcal{O} G) \rightarrow T\left(\mathcal{O}\left[N_{G}(P)\right]\right), \quad \alpha \mapsto \alpha(P),
$$

and it is well-known that this map restricts to a map

$$
T(\mathcal{O} G \sigma) \rightarrow T\left(\mathcal{O}\left[N_{G}(P)\right] \operatorname{br}_{P}(\sigma)\right)
$$

for every idempotent $\sigma$ of $Z(\mathcal{O} G)$; cf. also Lemma 2.2 for a proof. If $M$ is a trivial source $\mathcal{O} G$-module we denote by $M(P)$ any trivial source $\mathcal{O} N_{G}(P)$-module satisfying $M(P) / \mathfrak{p} M(P) \cong \bar{M}(P)$. Then $M(P)$ is uniquely determined up to isomorphism.

Note that if $\gamma \in T(\mathcal{O} G, \mathcal{O} H)=T(\mathcal{O}[G \times H])$ and $P \leqslant D$, then $\gamma(\Delta P) \in$ $T\left(\mathcal{O}\left[N_{G \times H}(\Delta P)\right]\right)$, and since $C_{G}(P) \times C_{H}(P) \leqslant N_{G \times H}(\Delta P)$, we may view $\gamma(\Delta P)$ 
as an element of $T\left(\mathcal{O}\left[C_{G}(P)\right], \mathcal{O}\left[C_{H}(P)\right]\right)$ via restriction. It follows from above that if $\gamma \in T(\mathcal{O} G \sigma, \mathcal{O} H \tau)$ for idempotents $\sigma$ (resp. $\tau$ ) of $Z(\mathcal{O} G)$ (resp. $Z(\mathcal{O} H)$ ), then $\gamma(\Delta P) \in T\left(\mathcal{O}\left[C_{G}(P)\right] \operatorname{br}_{P}(\sigma), \mathcal{O}\left[C_{H}(P)\right] \operatorname{br}_{P}(\tau)\right)$.

1.8. For an idempotent $\sigma$ of $Z(\mathcal{O} G)$ we denote by $R(K G \sigma)$ (resp. $R(k G \sigma)$ ) the Grothendieck group of finitely generated $K G \sigma$-modules (resp. $k G \sigma$-modules) and identify this group with the group of generalized characters $\chi$ (resp. Brauer characters $\lambda$ ) of $G$ satisfying $\chi(\sigma x)=\chi(x)$ for $x \in G$ (resp. $\lambda(\sigma y)=\lambda(y)$ for $y \in$ $G_{p^{\prime}}$, where $\lambda(a)$ is defined as $\sum_{x \in G_{p^{\prime}}} \alpha_{x} \lambda(x)$ for a Brauer character $\lambda$ and $a=$ $\sum_{x \in G} \alpha_{x} x \in \mathcal{O} G$ ). If $\sigma=1$, we just write $R(K G)$ (resp. $R(k G)$ ). The class of a $K G$-module (resp. $k G$-module) $V$ in $R(K G)$ (resp. $R(k G)$ ) will be denoted by $[[V]]$. Similarly, we identify $K \otimes R(K G)$ (resp. $K \otimes R(k G)$ ) with the $K$ vector space of class functions on $G$ (resp. on $G_{p^{\prime}}$ ) with values in $K$. If $\tau$ is an idempotent in $Z(\mathcal{O H})$ we denote by $R(K G \sigma, K H \tau)$ (resp. $R(k G \sigma, k H \tau)$ ) the Grothendieck groups of $(K G \sigma, K H \tau)$ - (resp. $(k G \sigma, k H \tau)$-) bimodules or the corresponding $K[G \times H]$-modules (resp. $k[G \times H]$-modules) under the identification from (1.1.a). For $\gamma \in T(\mathcal{O} G, \mathcal{O} H), \mu \in R(K G, K H)$, and $\lambda \in R(k G, k H)$ we define $\sigma \gamma \tau \in T(\mathcal{O} G \sigma, \mathcal{O} H \tau), \sigma \mu \tau \in R(K G \sigma, K H \tau)$, and $\sigma \lambda \tau \in R(k G \sigma, k H \tau)$ as induced by associating an $(\mathcal{O} G, \mathcal{O} H)$-bimodule $M$ to the $(\mathcal{O} G \sigma, \mathcal{O} H \tau)$-bimodule $\sigma M \tau$, and similarly over $K$ and $k$. We also define the maps introduced in (1.2.a) and (1.2.b) in the obvious way for character rings and Brauer character rings.

For a cyclic $p$-subgroup $P=\langle u\rangle$ of $G$ the generalized decomposition map $d_{G}^{u}$ is defined as

$$
\begin{aligned}
d_{G}^{u}: R(K G) & \rightarrow K \otimes R\left(k\left[C_{G}(P)\right]\right), \\
\chi & \mapsto(s \mapsto \chi(u s))
\end{aligned}
$$

for $s \in C_{G}(P)_{p^{\prime}}$. If $u=1$, then $d_{G}^{u}$ coincides with the usual decomposition map $d_{G}: R(K G) \rightarrow R(k G)$. Brauer's Second Main Theorem basically states that $d_{G}^{u}$ maps $R(K G \sigma)$ to $K \otimes R\left(k\left[C_{G}(P)\right] \operatorname{br}_{P}(\sigma)\right)$ for every central idempotent $\sigma$ of $\mathcal{O} G$. We will recover this result as a consequence of the approach presented in Section 2.

1.9. (a) Let $\sigma$ and $\tau$ be central idempotents of $\mathcal{O} G$ and $\mathcal{O} H$, respectively. In Br90] Broué defined a perfect isometry between $\mathcal{O} G \sigma$ and $\mathcal{O} H \tau$ as an element $\mu \in R(K G \sigma, K H \tau)$ satisfying the following conditions:

(i) $\mu(g, h) \in\left|C_{G}(g)\right| \mathcal{O} \cap\left|C_{H}(h)\right| \mathcal{O}$ for every $g \in G$ and $h \in H$;

(ii) If $g \in G$ and $h \in H$ such that $\mu(g, h) \neq 0$, then $g$ is a $p^{\prime}$-element if and only $h$ is a $p^{\prime}$-element;

(iii) The map $I_{\mu}: R(K H \tau) \rightarrow R(K G \sigma), \chi \mapsto \mu_{\dot{H}} \chi$, is an isometry (or equivalently, $\mu_{\dot{H}} \mu^{\check{ }}=[[K G \sigma]]$ and $\left.\mu_{\dot{G}}^{\check{ }} \mu=[[K H \tau]]\right)$.

If conditions (i) and (ii) are satisfied, $\mu$ is called perfect. If $\mu$ is perfect, then (by the Remark following [Br90, Prop. 1.3]) there exists a unique map $\tilde{I}_{\mu}: R(k H f) \rightarrow$ $R(k G e)$ satisfying $\tilde{I}_{\mu} \circ d_{H}=d_{G} \circ I_{\mu}$, or equivalently, $\tilde{I}_{\mu}(\lambda)=d_{G \times H}(\mu) \dot{H}_{H} \lambda$ for $\lambda \in R(k H f)$. If $\mu$ is a perfect isometry, then $\tilde{I}_{\mu}$ is an isomorphism.

(b) Next we adapt Broué's definition of an isotypy from Br90 to our situation and call it a global isotypy. A global isotypy between $\mathcal{O} G e$ and $\mathcal{O H f}$ is a family of perfect isometries $\mu_{P} \in R\left(K\left[C_{G}(P)\right] \operatorname{br}_{P}(e), K\left[C_{H}(P)\right] \operatorname{br}_{P}(f)\right), P \leqslant D$, such that 
the family of isometries

$$
I_{P}: R\left(K\left[C_{H}(P)\right] \operatorname{br}_{P}(f)\right) \rightarrow R\left(K\left[C_{G}(P)\right] \operatorname{br}_{P}(e)\right), \quad \chi \mapsto \mu_{P} \dot{C_{H}(P)} \underset{ }{\cdot} \chi,
$$

$P \leqslant D$, satisfies the following conditions:

(i) For every $P \leqslant D, g \in G$, and $h \in H$ such that $c_{g}=c_{h}: P \rightarrow D$, one has $I_{h_{P}}\left({ }^{h} \chi\right)={ }^{g} I_{P}(\chi)$.

(ii) For every $Q \leqslant D$ and every $p$-element $x \in C_{D}(Q)$ the diagram

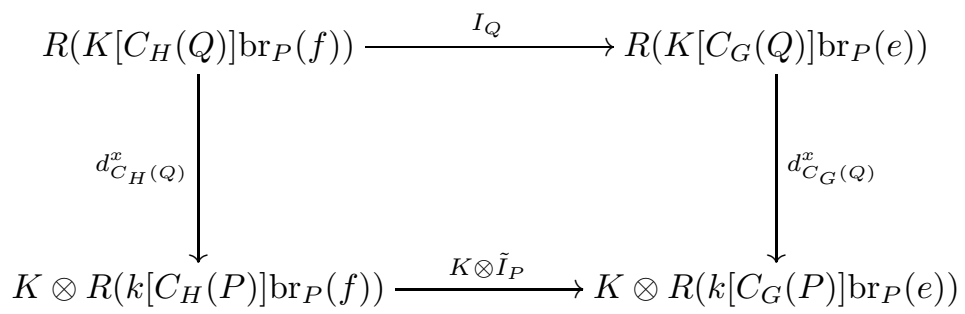

commutes, where $P$ denotes the subgroup of $D$ generated by $Q$ and $x$.

The above definition of a global isotypy is different from Broué's original definition in [Br90, Def. 4.6], since we do not require that the Brauer categories $\mathcal{B}_{f}(D, H)$ and $\mathcal{B}_{e}(D, G)$ are equivalent. We will see in the next lemma that this follows automatically, if the Frobenius categories are equivalent and $D$ is abelian. Also, we require isometries $I_{P}$ for every subgroup $P \leqslant D$, not only for the cyclic ones.

The following lemma makes the connection to the original definition of an isotypy clearer and will also be used in the proof of the main theorem.

1.10. Lemma. Let $\left(\mu_{P}\right)_{P \leqslant D}$, be a global isotypy between $\mathcal{O} G e$ and $\mathcal{O H f}$ as defined in Subsection 1.9(b). For every $P \leqslant D$, set

$$
\lambda_{P}:=d\left(\mu_{P}\right) \in R\left(k\left[C_{G}(P)\right] \operatorname{br}_{P}(e), k\left[C_{H}(P)\right] \operatorname{br}_{P}(f)\right) .
$$

Then there exists a bijection $\zeta$ between the objects of $\mathcal{B}_{f}(D, H)$ and $\mathcal{B}_{e}(D, G)$ preserving the first argument and having the following properties:

(i) For $P \leqslant D,(P, \varphi) \in \mathcal{B}_{f}(D, H)$ and $(P, \varepsilon) \in \mathcal{B}_{e}(D, G)$ one has

$$
\zeta(P, \varphi)=(P, \varepsilon) \Longleftrightarrow \varepsilon \mu_{P} \varphi \neq 0 \Longleftrightarrow \varepsilon \lambda_{P} \varphi \neq 0 .
$$

Moreover, if $\zeta(P, \varphi)=(P, \varepsilon)$, then $\varepsilon \mu_{P} \varphi$ is a perfect isometry between $\mathcal{O} C_{G}(P) \varepsilon$ and $\mathcal{O} C_{H}(P) \varphi$.

(ii) For $(P, \varphi) \in \mathcal{B}_{f}(D, H), g \in G$, and $h \in H$ such that $c_{g}=c_{h}: P \rightarrow D$, one has $\zeta\left({ }^{h}(P, \varphi)\right)={ }^{g} \zeta(P, \varphi)$. has

(iii) If $D$ is abelian, then, for $Q \leqslant P \leqslant D$ and $(P, \varphi),(Q, \psi) \in \mathcal{B}_{f}(D, H)$, one

$$
(Q, \psi) \leqslant(P, \varphi) \Longleftrightarrow \zeta(Q, \psi) \leqslant \zeta(P, \varphi) .
$$

(iv) If $D$ is abelian and $\operatorname{Hom}_{\mathcal{F}_{p}(G)}(Q, P)=\operatorname{Hom}_{\mathcal{F}_{p}(H)}(Q, P)$ for all p-subgroups $P$ and $Q$ of $D$, then

$$
\operatorname{Hom}_{\mathcal{B}_{e}(G)}(\zeta(Q, \psi), \zeta(P, \varphi))=\operatorname{Hom}_{\mathcal{B}_{f}(H)}((Q, \psi),(P, \varphi)),
$$

for all $(Q, \psi),(P, \varphi) \in \mathcal{B}_{f}(D, H)$. Moreover, the categories $\mathcal{B}_{e}(G)$ and $\mathcal{B}_{f}(H)$ are equivalent. 
Lemma 1.10 and the following Theorem 1.11 will be proved in Section 3. In the following theorem we prove several properties of $p$-permutation equivalences: Among others that (under additional hypotheses) they induce global isotypies and that via the Brauer construction they produce $p$-permutation equivalences on the local level. For the definition of the linear source ring $L(\mathcal{O} G)$ used in part (d) of the following theorem see Subsection 2.1

1.11. Theorem. Assume that $D$ is abelian and that

$$
\operatorname{Hom}_{\mathcal{F}_{p}(D, G)}(Q, P)=\operatorname{Hom}_{\mathcal{F}_{p}(D, H)}(Q, P)
$$

for all p-subgroups $P$ and $Q$ of $D$. Furthermore, let $\gamma \in T^{\Delta D}(\mathcal{O} G e, \mathcal{O} H f)$ be a p-permutation equivalence between $\mathcal{O} G e$ and $\mathcal{O} H f$. Then the following hold:

(a) For $P \leqslant D$ one has $\gamma(\Delta P) \in T^{\Delta D}\left(\mathcal{O}\left[C_{G}(P)\right] \operatorname{br}_{P}(e), \mathcal{O}\left[C_{H}(P)\right] \operatorname{br}_{P}(f)\right)$. In particular, $\gamma(\Delta P)$ is $\Delta D$-projective.

(b) For $P \leqslant D$, let $\mu_{P} \in R\left(K\left[C_{G}(P)\right] \operatorname{br}_{P}(e), K\left[C_{H}(P)\right] \operatorname{br}_{P}(f)\right)$ denote the generalized character of the element $\gamma(\Delta P)$. Then, $\mu_{P}$ is a perfect isometry between $\mathcal{O}\left[C_{G}(P)\right] \operatorname{br}_{P}(e)$ and $\mathcal{O}\left[C_{H}(P)\right] \operatorname{br}_{P}(f)$.

(c) For every $(P, \varphi) \in \mathcal{B}_{f}(D, H)$ there exists a unique element $(P, \varepsilon) \in \mathcal{B}_{e}(D, G)$ such that $\varepsilon \mu_{P} \varphi \neq 0$, and conversely for every $(P, \varepsilon) \in \mathcal{B}_{e}(D, G)$ there exists a unique $(P, \varphi) \in \mathcal{B}_{f}(D, H)$ such that $\varepsilon \mu_{P} \varphi \neq 0$. The resulting maps between the objects of $\mathcal{B}_{f}(D, H)$ and of $\mathcal{B}_{e}(D, G)$ are inverse bijections. In the sequel we denote the so-defined map $(P, \varphi) \mapsto(P, \varepsilon)$ by $\zeta$.

(d) Let $P \leqslant D, h \in H$ and $g \in G$ such that $c_{h}=c_{g}: P \rightarrow D$, and set $Q:=$ ${ }^{h} P={ }^{g} P$. Moreover, let $(P, \varphi) \in \mathcal{B}_{f}(D, H)$ and $(P, \varepsilon):=\zeta(P, \varphi) \in \mathcal{B}_{e}(D, G)$. Then $\zeta\left({ }^{h}(P, \varphi)\right)={ }^{g}(P, \varepsilon),{ }^{(g, h)} \gamma(\Delta P)=\gamma(\Delta Q)$, and

$$
{ }^{g}\left(\varepsilon \gamma(\Delta P) \varphi_{C_{H}(P)}^{\cdot} \alpha\right)=\left({ }^{g} \varepsilon\right) \gamma(\Delta Q)\left({ }^{h} \varphi\right) \underset{C_{H}(Q)}{\cdot}{ }^{h} \alpha
$$

for all $\alpha \in L\left(\mathcal{O}\left[C_{H}(P)\right] \operatorname{br}_{P}(\varphi)\right)$.

(e) The family $\left(\mu_{P}\right)_{P \leqslant D}$ is a global isotypy between $\mathcal{O} G e$ and $\mathcal{O H f}$ (in the sense of Subsection $1.9(\mathrm{~b}))$.

(f) Let $(P, \varphi),(Q, \psi) \in \mathcal{B}_{f}(D, H)$. Then one has $(Q, \psi) \leqslant(P, \varphi)$ if and only if $\zeta(Q, \psi) \leqslant \zeta(P, \varphi)$. Moreover, the categories $\mathcal{B}_{e}(G)$ and $\mathcal{B}_{f}(H)$ are equivalent.

(g) Let $P \leqslant D$ and let $\lambda_{P} \in R\left(k\left[C_{G}(P)\right] \operatorname{br}_{P}(e), k\left[C_{H}(P)\right] \operatorname{br}_{P}(f)\right)$ denote the generalized Brauer character of $\gamma(\Delta P)$. For $(P, \varphi) \in \mathcal{B}_{f}(D, H)$ and $(P, \varepsilon) \in \mathcal{B}_{e}(D, G)$, the following are equivalent:

(i) $\varepsilon \gamma(\Delta P) \varphi=0$.

(ii) $\varepsilon \mu_{P} \varphi=0$.

(iii) $\varepsilon \lambda_{P} \varphi=0$.

(h) Let $(P, \varphi) \in \mathcal{B}_{f}(D, H)$ and $\operatorname{set}(P, \varepsilon):=\zeta(P, \varphi) \in \mathcal{B}_{e}(D, H)$. Then, $\varepsilon \gamma(\Delta P) \varphi$ is a p-permutation equivalence between $\mathcal{O}\left[C_{G}(P)\right] \varepsilon$ and $\mathcal{O}\left[C_{H}(P)\right] \varphi$.

\section{The Brauer Construction And the Generalized DeCOMPOSITION MAP}

In this section we show that the Brauer construction on trivial source modules induces the generalized decomposition map on the level of Grothendieck groups; cf. Corollary 2.6. For later purposes we will need this result even for the generalized Brauer construction on linear source modules; cf. Theorem 2.4 Brauer's second main theorem will become a consequence of the property of the generalized Brauer construction in Lemma 2.2. For the reader's convenience we recall the relevant notions. 
2.1. Recall from Bo98 that a linear source $\mathcal{O} G$-module (or $p$-monomial $\mathcal{O} G$ module) $V$ is an $\mathcal{O}$-free $\mathcal{O} G$-module which is isomorphic to a direct summand of a monomial $\mathcal{O} G$-module (where the latter means a finite direct sum of modules of the form $\operatorname{Ind}_{H}^{G}\left(\mathcal{O}_{\varphi}\right)$, where $H \leqslant G, \varphi \in \operatorname{Hom}\left(H, \mathcal{O}^{\times}\right)$is a homomorphism into the unit group of $\mathcal{O}$, and $\mathcal{O}_{\varphi}$ denotes the $\mathcal{O} H$-module $\mathcal{O}$ with $H$-action given by $h \alpha=\varphi(h) \alpha$ for $h \in H$ and $\alpha \in \mathcal{O})$. Equivalently, the restriction of $V$ to a Sylow $p$-subgroup of $G$ is a monomial module, or also equivalently, every indecomposable direct summand of $V$ has a source of $\mathcal{O}$-rank 1. Every trivial source module is also a linear source module and the linear source ring $L(\mathcal{O} G)$, and its subgroups $L(\mathcal{O} G \sigma), L^{P}(\mathcal{O} G), L^{P}(\mathcal{O} G \sigma)$ are defined analogously to the terms $T(\mathcal{O} G)$, $T(\mathcal{O} G \sigma), T^{P}(\mathcal{O} G), T^{P}(\mathcal{O} G \sigma)$ for any $p$-subgroup $P$ of $G$ and any central idempotent $\sigma$ of $\mathcal{O} G$. Moreover, we view $T(\mathcal{O} G)$ as embedded in $L(\mathcal{O} G)$ in the natural way. Then also $T(\mathcal{O} G \sigma) \subseteq L(\mathcal{O} G \sigma)$, etc.

Let $\mathcal{M}_{G}^{p}$ denote the set of all pairs $(P, \varphi)$, where $P \leqslant G$ is a $p$-subgroup and $\varphi \in \operatorname{Hom}\left(P, \mathcal{O}^{\times}\right)$. Note that $G$ acts on $\mathcal{M}_{G}^{p}$ by conjugation: ${ }^{g}(P, \varphi):=\left({ }^{g} P,{ }^{g} \varphi\right)$ with $\left({ }^{g} \varphi\right)(x):=\varphi\left(g^{-1} x g\right)$ for $g \in G$ and $x \in{ }^{g} P$. We denote the $G$-stabilizer of $(P, \varphi)$ by $N_{G}(P, \varphi)$. Note that $P \leqslant N_{G}(P, \varphi)$. The set $\mathcal{M}_{G}^{p}$ is partially ordered by $(Q, \psi) \leqslant(P, \varphi): \Longleftrightarrow P \leqslant Q$ and $\psi=\left.\varphi\right|_{Q}$.

Recall from [BK00] that for any $\mathcal{O} G$-module $V$ and any $(P, \varphi) \in \mathcal{M}_{G}^{p}$ the generalized Brauer construction $\bar{V}(P, \varphi)$ is defined as

$$
\bar{V}(P, \varphi)=V^{(P, \varphi)} /\left(\sum_{R<P} \operatorname{tr}_{\left(R,\left.\varphi\right|_{R}\right)}^{(P, \varphi)}\left(V^{\left(R,\left.\varphi\right|_{R}\right)}\right)+\mathfrak{p} V^{(P, \varphi)}\right),
$$

where $V^{(P, \varphi)}:=\{v \in V \mid x v=\varphi(x) v$ for all $x \in P\}$ and $\operatorname{tr}_{(R, \psi)}^{(P, \varphi)}: V^{(R, \psi)} \rightarrow V^{(P, \varphi)}$ is defined by $v \mapsto \sum_{x R \in P / R} \varphi\left(x^{-1}\right) x v$ for $(P, \varphi),(R, \psi) \in \mathcal{M}_{G}^{p}$ with $R \leqslant P$ and $\psi=\left.\varphi\right|_{R}$ (see [BK00] for more details). The natural epimorphism $V^{(P, \varphi)} \rightarrow \bar{V}(P, \varphi)$ will be denoted by $\operatorname{Br}_{(P, \varphi)}^{V}$. Note that if $\varphi=1$, then we have $\bar{V}(P, 1)=\bar{V}(P)$ and $\operatorname{Br}_{(P, 1)}^{V}=\operatorname{Br}_{P}^{V}$; cf. Subsection 1.7 .

If $V$ is a linear source $\mathcal{O} G$-module and $(P, \varphi) \in \mathcal{M}_{G}^{p}$ then, by BK00, Theorem 3.3] (applied to $N_{G}(P, \varphi)$ as $G$ and $(P, \varphi)$ as $(H, \varphi)$ in their notation), there exists a decomposition

$$
\operatorname{Res}_{N_{G}(P, \varphi)}^{G}(V)=V^{\prime} \oplus V^{\prime \prime}
$$

into $\mathcal{O} N_{G}(P, \varphi)$-submodules $V^{\prime}$ and $V^{\prime \prime}$ with the property

$$
\operatorname{Res}_{P}^{N_{G}(P, \varphi)}\left(V^{\prime}\right) \cong \mathcal{O}_{\varphi} \oplus \cdots \oplus \mathcal{O}_{\varphi} \quad \text { and } \quad \mathcal{O}_{\varphi} \nmid \operatorname{Res}_{P}^{N_{G}(P, \varphi)}\left(V^{\prime \prime}\right) .
$$

Up to isomorphism, the module $V^{\prime}$ is uniquely determined by $V$ and $(P, \varphi)$, and we denote it by $V(P, \varphi)$. By [BK00, Theorem 3.5], the inclusion $V^{\prime} \rightarrow V$ induces an isomorphism between the reduction $V(P, \varphi) / \mathfrak{p} V(P, \varphi)$ and $\bar{V}(P, \varphi)$. If $V$ is a trivial source $\mathcal{O} G$-module and $\varphi=1$, then we have $V(P, 1) \cong V(P)$.

For every linear source $\mathcal{O} G$-module $V$, the $\mathcal{O}\left[N_{G}(P, \varphi)\right]$-module $V(P, \varphi)$ is again a linear source module and the $k\left[N_{G}(P, \varphi)\right]$-module $\bar{V}(P, \varphi)$ is a trivial source module. We obtain induced maps

$$
L(\mathcal{O} G) \rightarrow L\left(\mathcal{O}\left[N_{G}(P, \varphi)\right]\right), \quad[V] \mapsto[V(P, \varphi)],
$$

and

$$
L(\mathcal{O} G) \rightarrow T\left(k\left[N_{G}(P, \varphi)\right]\right), \quad[V] \mapsto[\bar{V}(P, \varphi)] .
$$


2.2. Lemma. Let $\sigma$ be a central idempotent of $\mathcal{O} G$, let $V$ be a linear source $\mathcal{O} G \sigma$ module, and let $(P, \varphi) \in \mathcal{M}_{G}^{p}$. Then, multiplication with $\operatorname{br}_{P}(\sigma) \in Z\left(\mathcal{O}\left[N_{G}(P, \varphi)\right]\right)$ is the identity map on $V(P, \varphi)$ and $\bar{V}(P, \varphi)$.

Proof. It suffices to show the statement for $\bar{V}(P, \varphi)$. So let $v \in V^{(P, \varphi)}$ and write $\sigma=\sum_{g \in G} \alpha_{g} g$ with $\alpha_{g} \in \mathcal{O}$ for $g \in G$. We have

$$
\begin{aligned}
\operatorname{Br}_{(P, \varphi)}^{V}(v)-\operatorname{br}_{P}(\sigma) \operatorname{Br}_{(P, \varphi)}^{V}(v) & =\operatorname{Br}_{(P, \varphi)}^{V}(\sigma v)-\operatorname{br}_{P}(\sigma) \operatorname{Br}_{(P, \varphi)}^{V}(v) \\
& =\operatorname{Br}_{(P, \varphi)}^{V}\left(\sum_{g \in G \backslash C_{G}(P)} \alpha_{g} g v\right),
\end{aligned}
$$

and it suffices to show that

$$
\operatorname{Br}_{(P, \varphi)}^{V}\left(\sum_{g \in \mathcal{C}} \alpha_{g} g v\right)=0
$$

for every $P$-conjugacy class $\mathcal{C}$ of $G$ containing an element $x \notin C_{G}(P)$. But in this case,

$$
\sum_{g \in \mathcal{C}} \alpha_{g} g v=\alpha_{x} \sum_{y \in P / C_{P}(x)} y x y^{-1} v=\operatorname{tr}_{\left(C_{P}(x),\left.\varphi\right|_{C_{P}(x)}\right.}^{(P, \varphi)}\left(\alpha_{x} x v\right)
$$

is a proper trace and the lemma is proved.

2.3. Next, let $P$ be a $p$-subgroup, let $u \in P$, and let $\zeta \in \mathcal{O}$ be a root of unity whose order is equal to the order of $u P^{\prime} \in P / P^{\prime}$, where $P^{\prime}$ denotes the derived subgroup of $P$. Then we have a map

$$
\begin{aligned}
-(P, u): L(\mathcal{O} G) & \rightarrow \mathbb{Z}[\zeta] \otimes L\left(\mathcal{O}\left[P C_{G}(P)\right]\right), \\
{[V] } & \mapsto \sum_{\varphi \in \operatorname{Hom}\left(P, \mathcal{O}^{\times}\right)} \varphi(u) \otimes \operatorname{res}_{P C_{G}(P)}^{N_{G}(P, \varphi)}([V(P, \varphi)]),
\end{aligned}
$$

which restricts to a map

$$
L(\mathcal{O} G \sigma) \rightarrow \mathbb{Z}[\zeta] \otimes L\left(\mathcal{O}\left[P C_{G}(P)\right] \operatorname{br}_{P}(\sigma)\right)
$$

for every central idempotent $\sigma$ of $\mathcal{O} G$, by Lemma 2.2 .

Note also that the diagram

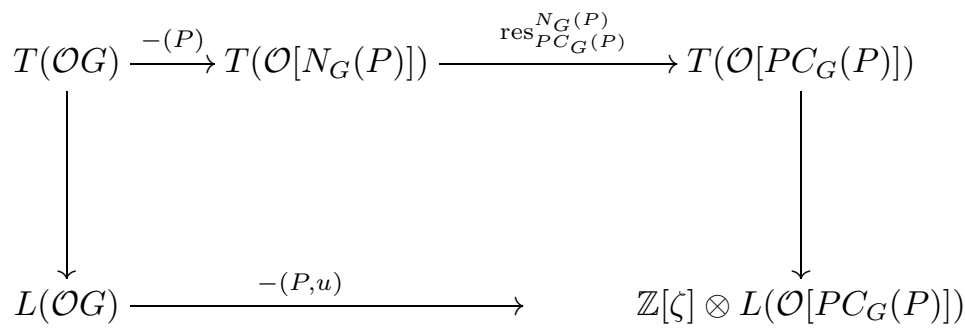

commutes for all $u \in P$, where the left vertical map is the inclusion map and the right vertical map maps the class $[V]$ to $1 \otimes[V]$ for every trivial source $\mathcal{O}\left[P C_{G}(P)\right]$ module $V$. In fact, for every trivial source $\mathcal{O} G$-module $V$ we have $V(P, \varphi)=0$ unless $\varphi=1$, and $V(P, 1) \cong V(P)$. 
Note that we have a commutative diagram

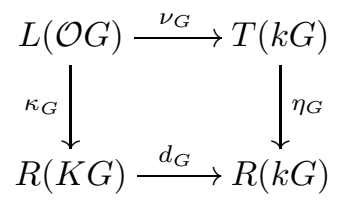

where $\kappa_{G}\left(\right.$ resp. $\left.\nu_{G}\right)$ is induced by tensoring a linear source $\mathcal{O} G$-module with $K$ (resp. $k$ ) over $\mathcal{O}$, and where $\eta_{G}$ is induced by taking the Brauer character of a trivial source $k G$-module. We will sometimes use the symbols $\kappa, \nu$, and $\eta$ without any index, if there is no risk of confusion. As a generalization of the above commutative diagram, we have the following theorem.

2.4. Theorem. Assume that $P=\langle u\rangle$ is a cyclic p-subgroup of $G$ and that $\zeta \in \mathcal{O}$ is a root of unity of the same order as $u$. Then the diagram

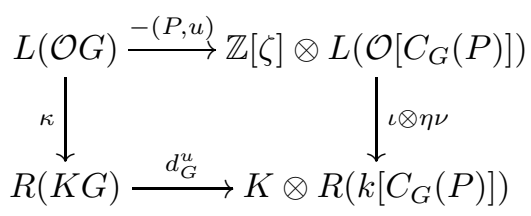

commutes, where $\iota$ denotes the inclusion $\mathbb{Z}[\zeta] \subset K$.

Proof. Let $s \in C_{G}(P)_{p^{\prime}}$ and consider the diagram

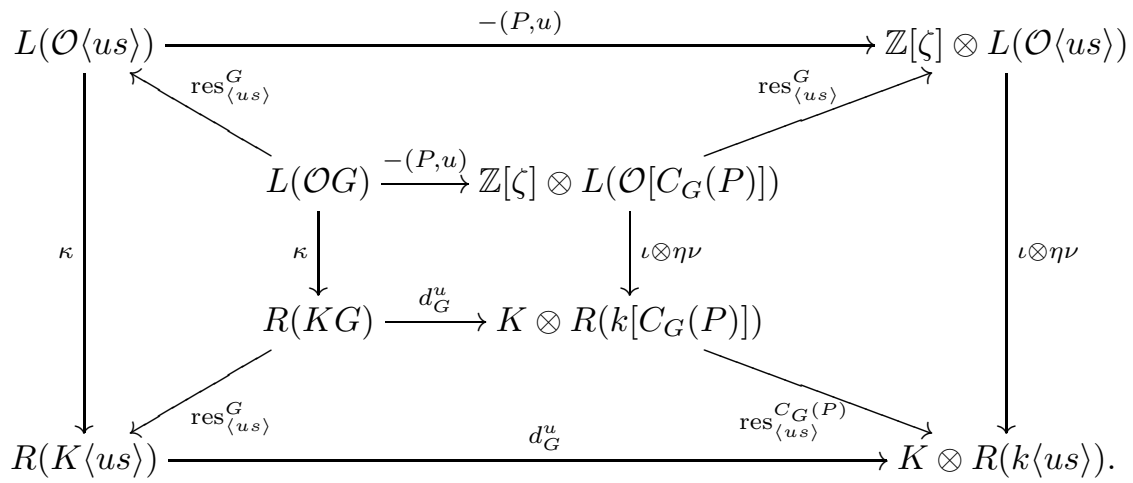

It is easy to verify that the outermost square commutes by noting that every indecomposable linear source $\mathcal{O}\langle u s\rangle$-module is of the form $\operatorname{Ind}_{H}^{\langle u s\rangle}\left(\mathcal{O}_{\psi}\right)$ for some subgroup $H$ of $\langle u s\rangle$ containing $s$. Moreover, the four squares surrounding the center square commute. In fact, this is trivial for the left, right, and bottom square, and for the top square it follows from the fact that $\operatorname{Res}_{\langle u s\rangle}^{N_{G}(P, \varphi)}(V(P, \varphi)) \cong$ $\left(\operatorname{Res}_{\langle u s\rangle}^{G}(V)\right)(P, \varphi)$ for all linear source $\mathcal{O} G$-modules $V$, as the defining property of $V(P, \varphi)$ shows. Since $s$ was arbitrary in $C_{G}(P)_{p^{\prime}}$ the lemma follows.

By the surjectivity of $\kappa_{G}: L(\mathcal{O} G) \rightarrow R(K G)$ and by (2.3.a), the previous theorem has the following immediate corollary which includes Brauer's Second Main Theorem.

2.5. Corollary. Assume the notation of Theorem 2.4, let $\sigma$ be a central idempotent of $\mathcal{O} G$, and let $\chi \in R(K G \sigma)$. Then $d_{G}^{u}(\chi) \in \mathbb{Z}[\zeta] \otimes R\left(k\left[C_{G}(P)\right] \operatorname{br}_{P}(\sigma)\right)$. 
For trivial source modules, Theorem 2.4 implies the following result.

2.6. Corollary. Assume the notation of Theorem 2.4 and let $\sigma$ be a central idempotent of $\mathcal{O} G$. Then the diagram

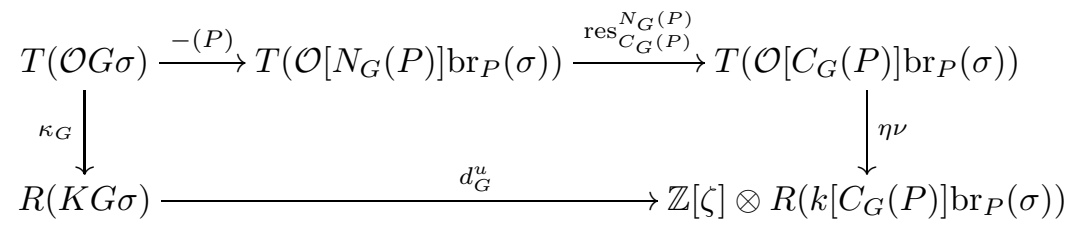

is commutative. In particular, $d_{G}^{u}(\chi) \in R\left(k\left[C_{G}(P)\right] \operatorname{br}_{P}(\sigma)\right)$ is a genuine Brauer character for every character $\chi$ of a trivial source $\mathcal{O} G \sigma$-module.

Proof. This follows immediately from Theorem 2.4, together with Lemma 2.2 and the commutativity of diagram (2.3.b).

By Corollary 2.6. the Brauer construction for a cyclic $p$-subgroup can be viewed as a lift of the generalized decomposition map. But the Brauer construction exists for all $p$-subgroups. That raises the question of whether there also exists a generalized decomposition map for arbitrary $p$-subgroups on the character level. The following proposition gives an answer to this question.

2.7. Proposition. For any p-subgroup $P$ of $G$ the following are equivalent:

(i) There exists a map $d_{G}^{P}: R(K G) \rightarrow K \otimes R\left(k\left[C_{G}(P)\right]\right)$ such that the diagram

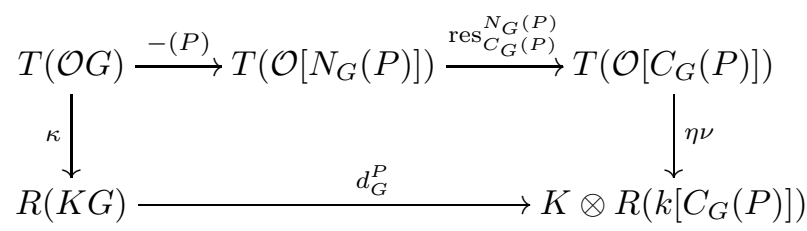

is commutative.

(ii) The group $P$ is cyclic.

Proof. If $P=\langle u\rangle$ is cyclic, then the diagram in (i) commutes for $d_{G}^{P}=d_{G}^{u}$ by Corollary 2.6 with $\sigma=1$. Conversely, assume that $P$ is not cyclic. We construct an element $x \in K \otimes T(\mathcal{O} G)$ with the property that

$$
\kappa_{G}(x)=0 \quad \text { and } \quad \eta_{C_{G}(P)} \nu_{C_{G}(P)}\left(\operatorname{res}_{C_{G}(P)}^{N_{G}(P)}(x(P))\right) \neq 0 .
$$

Recall that the species $s_{(Q, g)}: K \otimes T(\mathcal{O} G) \rightarrow K$ induce an isomorphism between the $K$-algebra $K \otimes T(\mathcal{O} G)$ and $K \times \cdots \times K$. Here, for a $p$-subgroup $Q$ of $G$, a $p^{\prime}$ element $g \in N_{G}(P)$, and a trivial source $\mathcal{O} G$-module $V$, the element $s_{(Q, g)}(V) \in K$ is defined as the Brauer character of $\bar{V}(Q)$ evaluated at $g$. Now, let $x$ be the element with $s_{(P, 1)}(x)=1$ and $s(x)=0$ for all other species of $T(\mathcal{O} G)$. Then, the image $\chi$ of $x$ in $K \otimes R(K G)$ is zero, since, viewing $\chi$ as a class function, one has $\chi(g)=s_{\left(\left\langle g_{p}\right\rangle, g_{p^{\prime}}\right)}(x)=0$ for all $g \in G$. On the other hand, since $s_{(P, 1)}(x)=1$ is the Brauer character value of $x(P)$ at the element 1 , the image $\lambda \in K \otimes R\left(k C_{G}(P)\right)$ of $x$, considered as a class function, has the property that $\lambda(1)=1$. 


\section{Proof of Lemma 1.10 and Theorem 1.11}

3.1. Proof of Lemma 1.10. Let $P \leqslant D$ and let $\operatorname{br}_{P}(e)=\varepsilon_{1}+\cdots+\varepsilon_{m}$ and $\operatorname{br}_{P}(f)=$ $\varphi_{1}+\cdots+\varphi_{n}$ be primitive idempotent decompositions in $Z\left(\mathcal{O}\left[C_{G}(P)\right]\right)$ and $Z\left(\mathcal{O}\left[C_{H}(P)\right]\right)$, respectively. Since $\mu_{P}$ is a perfect isometry between $\mathcal{O}\left[C_{G}(P)\right] \operatorname{br}_{P}(e)$ and $\mathcal{O}\left[C_{H}(P)\right] \operatorname{br}_{P}(f)$, Theorem 1.5(2) in $\underline{\operatorname{Br} 90}$ implies that $m=n$ and that, after renumbering if necessary, one has for $i \neq j$ in $\{1, \ldots, n\}$ :

$$
\varepsilon_{i} \mu_{P} \varphi_{j}=0, \quad \varepsilon_{i} \mu_{P} \varphi_{i} \neq 0, \quad \text { and } \quad \mu_{P}=\sum_{i=1}^{n} \varepsilon_{i} \mu_{P} \varphi_{i} .
$$

By applying the decomposition map, we obtain

$$
\varepsilon_{i} \lambda_{P} \varphi_{j}=0 \quad \text { and } \quad \lambda_{P}=\sum_{i=1}^{n} \varepsilon_{i} \lambda_{P} \varphi_{i}
$$

for $i \neq j$ in $\{1, \ldots, n\}$. Note that we also have

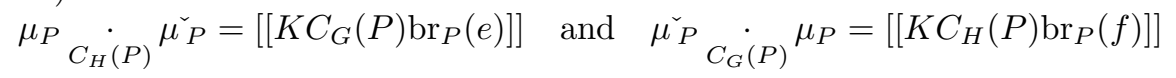

in $R\left(K\left[C_{G}(P)\right], K\left[C_{G}(P)\right]\right)$ and $R\left(K\left[C_{H}(P)\right], K\left[C_{H}(P)\right]\right)$, respectively, which implies

$$
\lambda_{P} \underset{C_{H}(P)}{\cdot} \lambda_{P}^{\check{P}}=\left[\left[k C_{G}(P) \operatorname{br}_{P}(e)\right]\right] \text { and } \quad \lambda_{P}^{\check{c}} \underset{C_{G}(P)}{.} \lambda_{P}=\left[\left[k C_{H}(P) \operatorname{br}_{P}(f)\right]\right]
$$

in $R\left(k\left[C_{G}(P)\right], k\left[C_{G}(P)\right]\right)$ and $R\left(k\left[C_{H}(P)\right], k\left[C_{H}(P)\right]\right)$. Multiplying the first equation in (3.1.C) by $\varepsilon_{i}$ from both sides and using (3.1.a) we obtain $\varepsilon_{i} \lambda_{P} \varphi_{i} \neq 0$ for all $i=1, \ldots, n$. Moreover, multiplying the first of the equations in 3.1.b by $\varepsilon_{i}$ and the second by $\varphi_{i}$ from both sides, we obtain

$$
\left(\varepsilon_{i} \mu_{P} \varphi_{i}\right)_{C_{H}(P)}\left(\varepsilon_{i} \mu_{P} \varphi_{i}\right)^{\swarrow}=\left[K C_{G}(P) \varepsilon_{i}\right]
$$

and

$$
\left(\varepsilon_{i} \mu_{P} \varphi_{i}\right)_{C_{G}(P)}^{\sim}\left(\varepsilon_{i} \mu_{P} \varphi_{i}\right)=\left[K C_{H}(P) \varphi_{i}\right]
$$

in $R\left(K\left[C_{G}(P)\right], K\left[C_{G}(P)\right]\right)$ and $R\left(K\left[C_{H}(P)\right], K\left[C_{H}(P)\right]\right)$, respectively. Moreover, it is easy to verify that the conditions of Proposition 4.1 in [Br90] are inherited from $\mu_{P}$ to $\varepsilon_{i} \mu_{P} \varphi_{i}$. Thus, $\varepsilon_{i} \mu_{P} \varphi_{i}$ is a perfect isometry between $\mathcal{O}\left[C_{G}(P)\right] \varepsilon_{i}$ and $\mathcal{O}\left[C_{H}(P)\right] \varphi_{i}$. Altogether we have established a bijection $\zeta:\left(P, \varphi_{i}\right) \mapsto\left(P, \varepsilon_{i}\right)$ between the objects of $\mathcal{B}_{f}(D, H)$ and $\mathcal{B}_{e}(D, G)$ satisfying condition (i) of Lemma 1.10 .

To establish property (ii), set $(P, \varepsilon):=\zeta(P, \varphi)$. Then it suffices to show that $\left({ }^{g} \varepsilon\right) \mu_{h_{P}}\left({ }^{h} \varphi\right) \neq 0$. But $\mu_{h_{P}}=(g, h) \mu_{P}$ so that $\left({ }^{g} \varepsilon\right) \mu_{h_{P}}\left({ }^{h} \varphi\right)={ }^{(g, h)}\left(\varepsilon \mu_{P} \varphi\right) \neq 0$.

Next, we establish property (iii). Set $(P, \varepsilon):=\zeta(P, \varphi)$ and $(Q, \delta):=\zeta(Q, \psi)$. Since, for every inclusion $(Q, \delta) \leqslant(P, \varepsilon)$ in $\mathcal{B}_{e}(D, G)$ and every intermediate group $Q \leqslant Q^{\prime} \leqslant P$ there exists a unique element $\left(Q^{\prime}, \delta^{\prime}\right) \in \mathcal{B}_{e}(D, G)$ such that $(Q, \delta) \leqslant$ $\left(Q^{\prime}, \delta^{\prime}\right) \leqslant(P, \varepsilon)$, we may assume that $P=\langle Q, x\rangle$ for some element $x \in P$. Note that $x \in C_{D}(Q)$, since $D$ is abelian, so that we can apply the commutative diagram in Subsection 1.9(b)(ii).

First assume that $(Q, \psi) \leqslant(P, \varphi)$ and let $\left(Q, \delta^{\prime}\right)$ be the unique element in $\mathcal{B}_{e}(D, G)$ satisfying $\left(Q, \delta^{\prime}\right) \leqslant(P, \varepsilon)$. Then $\varepsilon \operatorname{br}_{P}\left(\delta^{\prime}\right)=\varepsilon$ and $\varphi \operatorname{br}_{P}(\psi)=\varphi$. Since 
$\varepsilon \lambda_{P} \varphi_{C_{H}(P)}$ - is an isomorphism and since $d_{C_{H}(Q)}^{x}$, if $K$-linearly extended to a map $K \otimes R\left(K\left[C_{H}(Q)\right] \psi\right) \rightarrow K \otimes R\left(k\left[C_{H}(P)\right] \operatorname{br}_{P}(\psi)\right)$, is surjective, there exists $\chi \in R\left(K\left[C_{H}(Q)\right] \psi\right)$ such that $0 \neq d_{C_{H}(Q)}^{x}(\chi)=\varphi d_{C_{H}(Q)}^{x}(\chi)$, and we obtain

$$
\begin{aligned}
& 0 \neq\left(\varepsilon \lambda_{P} \varphi\right) \underset{C_{H}(P)}{\dot{\cdot}}\left(d_{C_{H}(Q)}^{x}(\chi)\right)=\varepsilon\left(\lambda_{P} \underset{C_{H}(P)}{\cdot}\left(\varphi d_{C_{H}(Q)}^{x}(\chi)\right)\right) \\
& =\varepsilon\left(\lambda_{P} \underset{C_{H}(P)}{\cdot} d_{C_{H}(Q)}^{x}(\psi \chi)\right)=\varepsilon\left(d_{C_{G}(Q)}^{x}\left(\lambda_{Q} \underset{C_{H}(Q)}{\cdot} \psi \chi\right)\right) \\
& =\varepsilon d_{C_{G}(Q)}^{x}\left(\delta^{\prime} \lambda_{Q} \psi_{C_{H}(Q)} \chi\right),
\end{aligned}
$$

using Brauer's Second Main Theorem or Corollary 2.5 for the last equality. This implies that $\delta^{\prime} \lambda_{Q} \psi \neq 0$ and $\delta^{\prime}=\delta$. Therefore, $(Q, \delta) \leqslant(P, \varepsilon)$. Similarly, one shows that $(Q, \delta) \leqslant(P, \varepsilon)$ implies $(Q, \psi) \leqslant(P, \varphi)$.

Finally, we establish property (iv). Let $a \in \operatorname{Hom}(Q, P)$ such that $a \in$ $\operatorname{Hom}_{\mathcal{B}_{f}(H)}((Q, \psi),(P, \varphi))$. Then there exists some $h \in H$ such that $a=c_{h}: Q \rightarrow P$ and ${ }^{h}(Q, \psi)=(P, \varphi)$. But we also have $a \in \operatorname{Hom}_{\mathcal{F}_{p}(H)}(Q, P)=\operatorname{Hom}_{\mathcal{F}_{p}(G)}(Q, P)$ and there exists some $g \in G$ such that $c_{h}=a=c_{g}: Q \rightarrow P$. Now, by the properties in (ii) and (iii), we obtain ${ }^{g} \zeta(Q, \psi)=\zeta\left({ }^{h}(Q, \psi)\right) \leqslant \zeta(P, \varphi)$. Thus, $a=c_{g}: Q \rightarrow P$ is also an element of $\operatorname{Hom}_{\mathcal{B}_{e}(G)}(\zeta(Q, \psi), \zeta(P, \varphi))$. Similarly, one shows the converse inclusion. Thus, $\zeta$ can be regarded as an isomorphism between the categories $\mathcal{B}_{f}(D, H)$ and $\mathcal{B}_{e}(D, G)$ which is the identity on morphism sets. Since $\mathcal{B}_{e}(D, G)$ contains an object from each isomorphism class of $\mathcal{B}_{e}(G)$, the inclusion $\mathcal{B}_{e}(D, G) \subseteq \mathcal{B}_{e}(G)$ is an equivalence. Similarly, the inclusion $\mathcal{B}_{f}(D, H) \subseteq \mathcal{B}_{f}(H)$ is an equivalence. Altogether, this implies that the categories $\mathcal{B}_{e}(G)$ and $\mathcal{B}_{f}(H)$ are equivalent.

For the proof of Theorem 1.11 we will need several lemmas which we will state and prove first.

For any two $p$-subgroups $P$ and $Q$ of $G$ we set

$$
T_{G}(Q, P):=\left\{g \in G \mid{ }^{g} Q \leqslant P\right\},
$$

and for any two subpairs $(Q, \delta)$ and $(P, \varepsilon)$ of $G$ we set

$$
T_{G}((Q, \delta),(P, \varepsilon)):=\left\{g \in G \mid{ }^{g}(Q, \delta) \leqslant(P, \varepsilon)\right\} .
$$

3.2. Lemma. Assume that $D$ is abelian and let $P \leqslant D$. Then one has

$$
T_{G}(P, D) \subseteq N_{G}(D) C_{G}(P) .
$$

Proof. Choose a Sylow e-subpair $(D, \varepsilon)$ and let $\delta$ be the unique primitive idempotent of $Z\left(\mathcal{O}\left[C_{G}(P)\right]\right)$ with $(P, \delta) \leqslant(D, \varepsilon)$. Let $g \in T_{G}(P, D)$. Then $\left({ }^{g} P,{ }^{g} \delta\right) \leqslant$ $\left(D, \varepsilon^{\prime}\right)$ for any primitive idempotent $\varepsilon^{\prime}$ of $Z\left(\mathcal{O}\left[C_{G}(D)\right]\right)$ occurring in $\operatorname{br}_{D}\left({ }^{g} \delta\right)$. By the Sylow Theorem for $e$-subpairs there exists $n \in N_{G}(D)$ such that ${ }^{n}\left(D, \varepsilon^{\prime}\right)=$ $(D, \varepsilon)$. This implies that $g=n^{-1}(n g)$ is contained in $N_{G}(D) \cdot T_{G}((P, \delta),(D, \varepsilon))$. Since $D$ is abelian, $N_{G}(D, \varepsilon)$ controls the fusion of subpairs contained in $(D, \varepsilon)$ (cf. AB79, Prop. 4.21]), i.e., $T_{G}((P, \delta),(D, \varepsilon)) \subseteq N_{G}(D, \varepsilon) C_{G}(P)$, and the result follows.

3.3. Lemma. Assume that $D$ is abelian and $\operatorname{Hom}_{\mathcal{F}_{p}(H)}(D, D) \subseteq \operatorname{Hom}_{\mathcal{F}_{p}(G)}(D, D)$ or $\operatorname{Hom}_{\mathcal{F}_{p}(G)}(D, D) \subseteq \operatorname{Hom}_{\mathcal{F}_{p}(H)}(D, D)$. Let $\gamma \in T^{\Delta D}(\mathcal{O} G, \mathcal{O} H)$, and let $P \leqslant D$. Then

$$
\gamma(\Delta P) \in T^{\Delta D}\left(\mathcal{O}\left[C_{G}(P)\right], \mathcal{O}\left[C_{H}(P)\right]\right) .
$$


Proof. By the Mackey decomposition formula and by standard facts about the Brauer construction we obtain

$$
\gamma(\Delta P) \in \sum_{\substack{(g, h) \in G \times H \\ \Delta P \leqslant{ }^{(g, h)} \Delta D}} T^{C_{G}(P) \times C_{H}(P) \cap^{(g, h)} \Delta D}\left(\mathcal{O}\left[C_{G}(P)\right], \mathcal{O}\left[C_{H}(P)\right]\right) .
$$

Now let $(g, h) \in G \times H$ with $\Delta P \leqslant{ }^{(g, h)} \Delta D$. Since $D$ is abelian and $P \leqslant{ }^{g} D$, we obtain ${ }^{g} D \leqslant C_{G}(P)$, and similarly, ${ }^{h} D \leqslant C_{H}(P)$. This implies

$$
C_{G}(P) \times C_{H}(P) \cap{ }^{(g, h)} \Delta D={ }^{(g, h)} \Delta D .
$$

Therefore, it suffices to show that ${ }^{(g, h)} \Delta D$ and $\Delta D$ are conjugate in $C_{G}(P) \times C_{H}(P)$. But $\Delta P \leqslant{ }^{(g, h)} \Delta D$ implies that $g^{-1} \in T_{G}(P, D), h^{-1} \in T_{H}(P, D)$, and $c_{g^{-1}}=$ $c_{h^{-1}}: P \rightarrow D$. By Lemma 3.2, there exist elements $m \in N_{G}(D), x \in C_{G}(P)$, $n \in N_{H}(D)$, and $y \in C_{H}(P)$ such that $g^{-1}=m x$ and $h^{-1}=n y$. This implies that

$$
c_{g^{-1}} \circ c_{x^{-1}}=c_{m}: D \rightarrow D \quad \text { and } \quad c_{h^{-1}} \circ c_{y^{-1}}=c_{n}: D \rightarrow D
$$

coincide on $P$. If $\operatorname{Hom}_{\mathcal{F}_{p}(H)}(D, D) \subseteq \operatorname{Hom}_{\mathcal{F}_{p}(G)}(D, D)$, then there exists $\tilde{m} \in$ $N_{G}(D)$ such that $c_{\tilde{m}}=c_{n}$ on $D$. This implies that $c_{\tilde{m}}, c_{m}$, and $c_{n}$ all coincide on $P$ so that $\tilde{m}^{-1} m \in C_{G}(P)$. Finally we obtain

$$
\Delta D={ }^{\left(\tilde{m}^{-1}, n^{-1}\right)} \Delta D={ }^{\left(\tilde{m}^{-1} m x, y\right)}\left({ }^{(g, h)} \Delta D\right)
$$

with $\tilde{m}^{-1} m x \in C_{G}(P)$ and $y \in C_{H}(P)$. If $\operatorname{Hom}_{\mathcal{F}_{p}(G)}(D, D) \subseteq \operatorname{Hom}_{\mathcal{F}_{p}(H)}(D, D)$ the proof is completed in a similar way.

3.4. For the next lemma we have to introduce the following notation. Let $L$ be a finite group and let $P$ be a common subgroup of $G, H$, and $L$. For $\varphi \in \operatorname{Hom}\left(P, \mathcal{O}^{\times}\right)$ we denote by $\Delta \varphi \in \operatorname{Hom}\left(\Delta P, \mathcal{O}^{\times}\right)$the homomorphism given by $\Delta \varphi(x, x)=\varphi(x)$ for $x \in P$. If $\psi \in \operatorname{Hom}\left(P, \mathcal{O}^{\times}\right)$and if $X$ is an $(\mathcal{O} G, \mathcal{O} H)$-bimodule, then $\bar{X}(\Delta P, \Delta \psi)$ is a $\left(k\left[C_{G}(P)\right], k\left[C_{H}(P)\right]\right)$-bimodule, since $C_{G}(P) \times C_{H}(P) \leqslant N_{G \times H}(\Delta P, \Delta \psi)$. Also, if $\lambda \in \operatorname{Hom}\left(P, \mathcal{O}^{\times}\right)$and if $Y$ is an $(\mathcal{O} H, \mathcal{O} L)$-bimodule, we may define the map

$$
\begin{aligned}
f_{P, \psi, \lambda}^{X, Y}: \bar{X}(\Delta P, \Delta \psi) \otimes_{k\left[C_{H}(P)\right]} \bar{Y}(\Delta P, \Delta \lambda) & \rightarrow\left(\overline{X \otimes_{\mathcal{O} H} Y}\right)(\Delta P, \Delta(\psi \lambda)), \\
\operatorname{Br}_{(\Delta P, \Delta \psi)}^{X}(x) \otimes \operatorname{Br}_{(\Delta P, \Delta \lambda)}^{Y}(y) & \mapsto \operatorname{Br}_{(\Delta P, \Delta(\psi \lambda))}^{X \otimes_{\mathcal{O} H} Y}(x \otimes y),
\end{aligned}
$$

for $x \in X^{(\Delta P, \Delta \psi)}$ and $y \in Y^{(\Delta P, \Delta \lambda)}$. We leave it to the reader to check that this map is well-defined, a homomorphism of $\left(k\left[C_{G}(P)\right], k\left[C_{L}(P)\right]\right)$-bimodules, and functorial in $X$ and $Y$. Next we fix $\varphi \in \operatorname{Hom}\left(P, \mathcal{O}^{\times}\right)$and define the map

$$
f_{P, \varphi}^{X, Y}: \bigoplus_{\substack{\psi, \lambda \in \operatorname{Hom}(P, \mathcal{O} \times) \\ \psi \lambda=\varphi}} \bar{X}(\Delta P, \Delta \psi) \otimes_{k\left[C_{H}(P)\right]} \bar{Y}(\Delta P, \Delta \lambda) \rightarrow\left(\overline{X \otimes_{\mathcal{O} H} Y}\right)(\Delta P, \Delta \varphi)
$$

as the sum of the maps $f_{P, \psi, \lambda}^{X, Y}$.

If $Y$ is just a left $\mathcal{O} H$-module, we may regard it as an $(\mathcal{O} H, \mathcal{O} L)$-bimodule, denoted by $Y_{L}$, with the trivial right $L$-action for any group $L$. If $L$ contains $P$, then $Y_{L}^{(\Delta Q, \Delta \lambda)}=Y^{(Q, \lambda)}$ and $\operatorname{tr}_{(\Delta Q, \Delta \lambda \mid \Delta Q)}^{(\Delta P, \Delta \lambda)}: Y_{L}^{(\Delta Q, \Delta \lambda \mid \Delta Q)} \rightarrow Y_{L}^{(\Delta P, \Delta \lambda)}$ coincides with $\operatorname{tr}_{\left(Q,\left.\lambda\right|_{Q}\right)}^{(P, \lambda)}: Y^{\left(Q,\left.\lambda\right|_{Q}\right)} \rightarrow Y^{(P, \lambda)}$ for every $\lambda \in \operatorname{Hom}\left(P, \mathcal{O}^{\times}\right)$and every $Q \leqslant P$. 
This implies that $\bar{Y}_{L}(\Delta P, \Delta \lambda)=\bar{Y}(P, \lambda)$, and we obtain for $\psi, \lambda \in \operatorname{Hom}\left(P, \mathcal{O}^{\times}\right)$ (as a special case of $\left.f_{P, \psi, \lambda}^{X, Y}\right)$ a well-defined $k\left[C_{G}(P)\right]$-module homomorphism

$$
\begin{aligned}
g_{P, \psi, \lambda}^{X, Y}: \bar{X}(\Delta P, \Delta \psi) \otimes_{k\left[C_{H}(P)\right]} \bar{Y}(P, \lambda) & \rightarrow\left(\overline{X \otimes_{\mathcal{O} H} Y}\right)(P, \psi \lambda), \\
\operatorname{Br}_{(\Delta P, \Delta \psi)}^{X}(x) \otimes \operatorname{Br}_{(P, \lambda)}^{Y}(y) & \mapsto \operatorname{Br}_{(P, \psi \lambda)}^{X \otimes_{\mathcal{O} H} Y}(x \otimes y),
\end{aligned}
$$

with $x \in X^{(\Delta P, \Delta \psi)}$ and $y \in Y^{(P, \lambda)}$, which is natural in $X$ and $Y$. Again, for $\varphi \in \operatorname{Hom}\left(P, \mathcal{O}^{\times}\right)$(as a special case of $f_{P, \varphi}^{X, Y}$ ) we obtain a well-defined $k\left[C_{G}(P)\right]$ module homomorphism

$$
g_{P, \varphi}^{X, Y}: \bigoplus_{\substack{\psi, \lambda \in \operatorname{Hom}\left(P, \mathcal{O}^{\times}\right) \\ \psi \lambda=\varphi}} \bar{X}(\Delta P, \Delta \psi) \otimes_{k\left[C_{H}(P)\right]} \bar{Y}(P, \lambda) \rightarrow\left(\overline{X \otimes_{\mathcal{O} H} Y}\right)(P, \psi \lambda),
$$

as the sum of the maps $g_{P, \psi, \lambda}^{X, Y}$, which is functorial in $X$ and $Y$.

3.5. Lemma. Assume the notation from Subsection 3.4 and let $Q$ be a common subgroup of $G$ and $H$. Furthermore, assume that $\mathcal{R} \subseteq G$ is a set of representatives of $G / Q$ and set $\mathcal{R}^{\prime}:=\left\{g \in \mathcal{R} \mid P \leqslant{ }^{g} Q\right\}$. Finally, assume that for every $g \in \mathcal{R}^{\prime}$ there exists an element $h_{g} \in H$ such that $c_{g^{-1}}=c_{h_{g}^{-1}}: P \rightarrow Q$. If $X=\operatorname{Ind}_{\Delta Q}^{G \times H}\left(\mathcal{O}_{\Delta \theta}\right)=$ $\mathcal{O}[G \times H] \otimes_{\mathcal{O}_{\Delta Q}} \mathcal{O}_{\Delta \theta}$ for some $\theta \in \operatorname{Hom}\left(Q, \mathcal{O}^{\times}\right)$, then the following hold:

(a) The module $X$ is a free right $\mathcal{O} H$-module with basis $(g, 1) \otimes 1, g \in \mathcal{R}$.

(b) For $\psi \in \operatorname{Hom}\left(P, \mathcal{O}^{\times}\right)$, the module $\bar{X}(\Delta P, \Delta \psi)$ is a free right $k\left[C_{H}(P)\right]$ module with basis $\operatorname{Br}_{(\Delta P, \Delta \psi)}^{X}\left(\left(g, h_{g}\right) \otimes 1\right)$, where $g$ runs through all elements of $\mathcal{R}^{\prime}$ satisfying $(P, \psi) \leqslant{ }^{g}(Q, \theta)$.

(c) The map $f_{P, \varphi}^{X, Y}$ is an isomorphism for every $(\mathcal{O} H, \mathcal{O} L)$-bimodule $Y$ and every $\varphi \in \operatorname{Hom}\left(P, \mathcal{O}^{\times}\right)$.

(d) The map $g_{P, \varphi}^{X, Y}$ is an isomorphism for every left $\mathcal{O} H$-module $Y$ and every $\varphi \in \operatorname{Hom}\left(P, \mathcal{O}^{\times}\right)$.

Proof. (a) It is easy to check that $\mathcal{R} \times H$ is a set of representatives for $(G \times H) / \Delta Q$. Thus, $(G \times H) / \Delta Q$ is a free left $H$-set with $H$-orbit representatives $(g, 1), g \in \mathcal{R}$. Now the result is immediate.

(b) Since $\mathcal{R} \times H$ is a set of representatives for $(G \times H) / \Delta Q$, we obtain from BK00, Proposition 2.4] that the elements $\operatorname{Br}_{(\Delta P, \Delta \psi)}^{X}((g, h) \otimes 1)$, with $(g, h) \in \mathcal{R} \times$ $H$ satisfying $(\Delta P, \Delta \psi) \leqslant{ }^{(g, h)}(\Delta Q, \Delta \theta)$, form a $k$-basis of $\bar{X}(\Delta P, \Delta \psi)$. But for $(g, h) \in \mathcal{R} \times H$ we have $(\Delta P, \Delta \psi) \leqslant{ }^{(g, h)}(\Delta Q, \Delta \theta)$ if and only if $(P, \psi) \leqslant{ }^{g}(Q, \theta)$ and $c_{g^{-1}}=c_{h^{-1}}: P \rightarrow Q$. Moreover, for $g \in \mathcal{R}^{\prime}$, the condition $c_{g^{-1}}=c_{h^{-1}}: P \rightarrow Q$ is equivalent to $h=c h_{g}$ for some $c \in C_{H}(P)$. Also, for any element $c \in C_{H}(P)$, we have $(1, c) \cdot \operatorname{Br}_{(\Delta P, \Delta \psi)}^{X}((g, h) \otimes 1)=\operatorname{Br}_{(\Delta P, \Delta \psi)}^{X}((g, c h) \otimes 1)$. This, together with part (a), proves part (b).

(c) Using part (b) we can identify the domain of the map $f_{P, \varphi}^{X, Y}$ by means of the $k$-linear isomorphism

$$
\begin{gathered}
\kappa: \bigoplus_{g \in \mathcal{R}^{\prime}} \bar{Y}\left(\Delta P, \Delta \lambda_{g}\right) \rightarrow \bigoplus_{\substack{\psi, \lambda \in \operatorname{Hom}(P, \mathcal{O}) \\
\psi \lambda=\varphi}} \bar{X}(\Delta P, \Delta \psi) \otimes_{k\left[C_{H}(P)\right]} \bar{Y}(\Delta P, \Delta \lambda), \\
\left(\operatorname{Br}_{\left(\Delta P, \Delta \lambda_{g}\right)}^{Y}\left(y_{g}\right)\right)_{g \in \mathcal{R}^{\prime}} \mapsto \sum_{g \in \mathcal{R}^{\prime}} \operatorname{Br}_{\left(\Delta P, \Delta \psi_{g}\right)}^{X}\left(\left(g, h_{g}\right) \otimes 1\right) \otimes \operatorname{Br}_{\left(\Delta P, \Delta \lambda_{g}\right)}^{Y}\left(y_{g}\right),
\end{gathered}
$$


where, for $g \in \mathcal{R}^{\prime}$, the homomorphism $\psi_{g}$ is defined as the restriction of ${ }^{g} \theta$ to $P$ and $\lambda_{g}$ is defined as $\varphi \psi_{g}^{-1}$.

Next we want to describe the range of $f_{P, \varphi}^{X, Y}$. First it is easy to check that the map

$$
\begin{aligned}
\mu: \operatorname{Ind}_{\Delta Q}^{G \times H}\left(\mathcal{O}_{\theta}\right) \otimes_{\mathcal{O} H} Y & \rightarrow \mathcal{O} G \otimes_{\mathcal{O} Q}\left(\mathcal{O}_{\theta \times 1} \otimes \operatorname{Res}_{Q \times L}^{H \times L}(Y)\right), \\
((g, h) \otimes 1) \otimes y & \mapsto g \otimes\left(1 \otimes h^{-1} y\right),
\end{aligned}
$$

is a well-defined isomorphism of $(\mathcal{O} G, \mathcal{O} L)$-bimodules with inverse $(g \otimes(1 \otimes y)) \mapsto$ $((g, 1) \otimes 1) \otimes y$ for $g \in G$ and $y \in Y$. Here, $\mathcal{O}_{\theta \times 1}$ is the left $\mathcal{O}[Q \times L]$-module $\mathcal{O}$ with $(q, l) \cdot \alpha=\theta(q) \alpha$ for $\alpha \in \mathcal{O}$ and $(q, l) \in Q \times L$. Moreover, since $P \times L$ contains $\Delta P$, we may compute $\left(\overline{X \otimes_{\mathcal{O} H} Y}\right)(P, \varphi)$ as a $k$-vector space by first restricting the image of $\mu$ to $P \times L$. By Mackey, we have an isomorphism

$$
\begin{gathered}
\nu: \bigoplus_{g \in \mathcal{R}^{\prime \prime}} \mathcal{O} P \otimes_{\mathcal{O}\left[P \cap{ }^{g} Q\right]}\left(\mathcal{O}_{\theta \times 1} \otimes{ }^{g} Y\right) \rightarrow \mathcal{O} G \otimes \mathcal{O Q}\left(\mathcal{O}_{\theta \times 1} \otimes \operatorname{Res}_{Q \times L}^{H \times L}(Y)\right), \\
\left(a_{g} \otimes\left(\alpha \otimes y_{g}\right)\right)_{g \in \mathcal{R}^{\prime \prime}} \mapsto \sum_{g \in \mathcal{R}^{\prime \prime}} a_{g} g \otimes\left(\alpha \otimes y_{g}\right),
\end{gathered}
$$

of $(\mathcal{O} P, \mathcal{O} L)$-bimodules, where $\mathcal{R}^{\prime \prime} \subseteq \mathcal{R}$ is a set of representatives for the double cosets $P \backslash G / Q$, and where for $g \in \mathcal{R}^{\prime \prime}$, ${ }^{g} Y$ denotes the right $\mathcal{O} L$-module $Y$ with a left $\mathcal{O}\left[P \cap{ }^{g} Q\right]$-module structure $a * g y:=g^{-1} a g y$ for $a \in P \cap{ }^{g} Q$ and $y \in Y$. Note that $\mathcal{R}^{\prime} \subseteq \mathcal{R}^{\prime \prime}$, since if $g \in \mathcal{R}^{\prime}$, then $P \leqslant{ }^{g} Q$ implies $P g Q=g Q$. Moreover, note that for $g \in \mathcal{R}^{\prime \prime}$ one has $P \cap{ }^{g} Q=P$ if and only if $g \in \mathcal{R}^{\prime}$. Next, we show that for $g \in \mathcal{R}^{\prime \prime} \backslash \mathcal{R}^{\prime}$ one has

$$
\left(\overline{\mathcal{O} P \otimes_{\mathcal{O}\left[P \cap{ }^{g} Q\right]}{ }^{g} Y^{\prime}}\right)(\Delta P, \Delta \varphi)=0,
$$

where ${ }^{g} Y^{\prime}$ denotes the $\left(\mathcal{O}\left[P \cap{ }^{g} Q\right], \mathcal{O} L\right)$-bimodule $\mathcal{O}_{g_{\theta \times 1}} \otimes{ }^{g} Y$. In fact, let $\mathcal{S}$ denote a set of representatives of $P / R$ containing 1 , where $R:=P \cap{ }^{g} Q<P$, and assume that $y=\sum_{a \in \mathcal{S}} a \otimes y_{a}^{\prime} \in\left(\mathcal{O} P \otimes_{\mathcal{O} R}{ }^{g} Y^{\prime}\right)^{(\Delta P, \Delta \varphi)}$. Then, for every $r \in R$ we obtain

$$
\sum_{a \in \mathcal{S}} a \otimes \varphi(r) y_{a}^{\prime}=\varphi(r) y=(r, r) \cdot y=\sum_{a \in \mathcal{S}} r a \otimes y_{a}^{\prime} r^{-1},
$$

and comparing summands with respect to the elements $a \in \mathcal{S}$ in the first factor we obtain $\varphi(r) y_{1}^{\prime}=r y_{1}^{\prime} r^{-1}$. Thus, $1 \otimes y_{1}^{\prime} \in\left(\mathcal{O} P \otimes_{\mathcal{O} R}{ }^{g} Y^{\prime}\right)^{(R, \varphi)}$. Moreover, for every $a_{0} \in \mathcal{S}$, we obtain

$$
\sum_{a \in \mathcal{S}} a \otimes \varphi\left(a_{0}\right) y_{a}^{\prime}=\varphi\left(a_{0}\right) y=\left(a_{0}, a_{0}\right) \cdot y=\sum_{a \in \mathcal{S}} a_{0} a \otimes y_{a}^{\prime} a_{0}^{-1}
$$

By comparing summands with first factor $a_{0}$ we obtain $\varphi\left(a_{0}\right) y_{a_{0}}^{\prime}=y_{1}^{\prime} a_{0}^{-1}$. This implies that

$$
y=\sum_{a \in \mathcal{S}} a \otimes y_{a}^{\prime}=\sum_{a \in \mathcal{S}} a \otimes \varphi(a)^{-1} y_{1}^{\prime} a^{-1}=\operatorname{tr}_{\left(R,\left.\varphi\right|_{R}\right)}^{(P, \varphi)}\left(1 \otimes y_{1}^{\prime}\right),
$$

and the claim in (3.5.al) is proved. 
Combining the isomorphisms $\mu$ and $\nu$ we obtain the $k$-linear isomorphism $\omega:=$ $\left(\overline{\mu^{-1} \circ \nu}\right)(\Delta P, \Delta \varphi)$ which is given explicitly by

$$
\begin{aligned}
& \omega: \bigoplus_{g \in \mathcal{R}^{\prime}}\left(\overline{{ }^{g} Y^{\prime}}\right)(\Delta P, \Delta \varphi) \rightarrow\left(\overline{X \otimes_{\mathcal{O} H} Y}\right)(\Delta P, \Delta \varphi), \\
& \left(\operatorname{Br}_{(\Delta P, \Delta \varphi)}^{g_{Y^{\prime}}}\left(1 \otimes y_{g}\right)\right) \mapsto \sum_{g \in \mathcal{R}^{\prime}} \operatorname{Br}_{(\Delta P, \Delta \varphi)}^{X \otimes_{\mathcal{O} H} Y}\left(((g, 1) \otimes 1) \otimes y_{g}\right),
\end{aligned}
$$

for $y_{g} \in{ }^{g} Y^{\prime}$. Note that the composition

$$
\omega^{-1} \circ f_{P, \varphi}^{X, Y} \circ \kappa: \bigoplus_{g \in \mathcal{R}^{\prime}} \bar{Y}\left(\Delta P, \Delta \lambda_{g}\right) \rightarrow \bigoplus_{g \in \mathcal{R}^{\prime}}\left(\overline{{ }^{g} Y^{\prime}}\right)(\Delta P, \Delta \varphi)
$$

is given by the component-wise map

$$
\left(\operatorname{Br}_{\left(\Delta P, \Delta \lambda_{g}\right)}^{Y}\left(y_{g}\right)\right)_{g \in \mathcal{R}^{\prime}} \mapsto\left(\operatorname{Br}_{(\Delta P, \Delta \varphi)}^{g_{Y^{\prime}}}\left(1 \otimes h_{g}^{-1} y_{g}\right)\right)_{g \in \mathcal{R}^{\prime}}
$$

which is a $k$-linear isomorphism. In fact, for $g \in \mathcal{R}^{\prime}$ we had ${ }^{g} Y^{\prime}=\mathcal{O}_{g_{\theta \times 1}} \otimes{ }^{g} Y$, and it is now easy to check that $y \mapsto h_{g}^{-1} y$ induces an isomorphism $\bar{Y}\left(\Delta P, \Delta \lambda_{g}\right) \rightarrow$ $\left(\overline{{ }^{g_{Y}}}\right)\left(\Delta P, \Delta \lambda_{g}\right)$ and that $y \mapsto 1 \otimes y$ induces an isomorphism $\left({ }^{{ }^{\bar{Y}}}\right)\left(\Delta P, \Delta \lambda_{g}\right) \rightarrow$ $\left({ }^{g} Y^{\prime}\right)(\Delta P, \Delta \varphi)$. Therefore, with $\omega$ and $\kappa, f_{P, \varphi}^{X, Y}$ is also an isomorphism.

(d) This is a special case of part (c) by the second part of Subsection 3.4

3.6. Corollary. Let $G, H, L, P$, and $\varphi$ be as in Subsection 3.4 and let $Q$ be a common p-subgroup of $G$ and $H$. Moreover let $X$ be a relatively $\Delta Q$-projective linear source $(\mathcal{O} G, \mathcal{O} H)$-bimodule. Finally, assume that $\operatorname{Hom}_{\mathcal{F}_{p}(G)}(P, R) \subseteq$ $\operatorname{Hom}_{\mathcal{F}_{p}(H)}(P, R)$ for every $R \leqslant Q$. Then the following hold:

(a) The map $f_{P, \varphi}^{X, Y}$ is an isomorphism for every $(\mathcal{O} H, \mathcal{O} L)$-bimodule $Y$, and the map $g_{P, \varphi}^{X, Y}$ is an isomorphism for every left $\mathcal{O} H$-module $Y$.

(b) If $X$ is a $\Delta Q$-projective trivial source $(\mathcal{O} G, \mathcal{O} H)$-bimodule, then the map $f_{P, 1, \varphi}^{X, Y}$ is an isomorphism for every $(\mathcal{O} H, \mathcal{O} L)$-bimodule $Y$ and the $\operatorname{map}_{P, 1, \varphi}^{X, Y}$ is an isomorphism for every left $\mathcal{O} H$-module $Y$. In particular one has an isomorphism of $\left(k\left[C_{G}(P)\right], k\left[C_{L}(P)\right]\right.$-bimodules,

$$
\bar{X}(\Delta P) \otimes_{k\left[C_{H}(P)\right]} \bar{Y}(\Delta P) \cong\left(\overline{X \otimes_{\mathcal{O} H} Y}\right)(\Delta P),
$$

for every $(\mathcal{O} H, \mathcal{O} L)$-bimodule $Y$ and an isomorphism of left $k\left[C_{G}(P)\right]$-modules,

$$
\bar{X}(\Delta P) \otimes_{k\left[C_{H}(P)\right]} \bar{Y}(P) \cong\left(\overline{X \otimes_{\mathcal{O} H} Y}\right)(P),
$$

for every left $\mathcal{O} H$-module $Y$.

Proof. (a) The functors $\bar{?}(\Delta P, \Delta \psi) \otimes_{k\left[C_{H}(P)\right]} \bar{Y}(\Delta P, \Delta \lambda)$ and $\left(\overline{?_{\otimes_{\mathcal{O} H} Y}}\right)(\Delta P, \Delta(\psi \lambda))$ are additive, and since $X$ is relatively $\Delta Q$-projective, $X$ is isomorphic to a direct summand of a direct sum of $(\mathcal{O} G, \mathcal{O} H)$-bimodules of the form $X^{\prime}=\operatorname{Ind}_{\Delta R}^{G \times H}\left(\mathcal{O}_{\Delta \theta}\right)$ for various $(R, \theta) \in \mathcal{M}_{Q}^{p}$. Since the natural transformation $f_{P, \varphi}^{X^{\prime}, Y}$ is an isomorphism for each $X^{\prime}$ by Lemma 3.5, $f_{P, \varphi}^{X, Y}$ is also an isomorphism. The statement concerning $g_{P, \varphi}^{X, Y}$ follows as a special case as explained in the second part of Subsection 3.4

(b) If $X$ is a trivial source $(\mathcal{O} G, \mathcal{O} H)$-bimodule, then $\bar{X}(\Delta P, \Delta \psi)=0$ for all $\psi \neq 1$ and the result follows immediately from part (a). 
3.7. Now we are ready to prove Theorem 1.11, The following two arguments will be used repeatedly in the proof.

(a) For any $\alpha \in T(\mathcal{O} G)$ and any $p$-subgroups $P \unlhd Q \leqslant G$ one has $\alpha(P)(Q)=$ $\operatorname{res}_{N_{G}(P) \cap N_{G}(Q)}^{N_{G}(Q)} \alpha(Q)$ in $T\left[\mathcal{O}\left[N_{G}(P) \cap N_{G}(Q)\right]\right.$; cf. [BK02, Lemma 1.10(vi)] for instance. This implies that if $P$ and $Q$ are $p$-subgroups of $G$ such that $Q \leqslant N_{G}(P)$, then $\alpha(P)(Q)=\alpha(P)(P Q)=\alpha(P Q)$ in $T\left(\mathcal{O}\left[N_{G}(P) \cap N_{G}(Q)\right]\right)$, for every $\alpha \in$ $T(\mathcal{O} G)$.

(b) If, for elements $\alpha, \beta \in T^{P}(\mathcal{O} G)$, the generalized Brauer characters of $\alpha(Q)$ and $\beta(Q)$ coincide for all $Q \leqslant P$, then $\alpha=\beta$. In fact, in this case all the species of $T(\mathcal{O} G)$ coincide on $\alpha$ and $\beta$. For more details see [Be84, Section 2.13] for example.

3.8. Proof of Theorem 1.11, (a) This follows from Lemma 3.3.

(b) Write $\gamma=[M]-[N]$, where $M$ and $N$ are $\Delta D$-projective $p$-permutation $(\mathcal{O} G e, \mathcal{O} H f)$-bimodules. Then, by Corollary 3.6(b), we have

$$
\begin{aligned}
& \nu\left(\gamma(\Delta P)_{C_{H}(P)}^{\dot{\cdot}} \gamma(\Delta P)^{\gamma}\right)=\bar{\gamma}(\Delta P)_{C_{H}(P)} \cdot \vec{\gamma}(\Delta P) \\
& =([\bar{M}(\Delta P)]-[\bar{N}(\Delta P)])_{C_{H}(P)}\left([\bar{M}(\Delta P)]-\left[\overline{N^{\top}}(\Delta P)\right]\right) \\
& =\left[\left(\overline{M \otimes_{\mathcal{O O H}} M}\right)(\Delta P)\right]-\left[\left(\overline{M \otimes_{\mathcal{O} H} N^{\top}}\right)(\Delta P)\right] \\
& -\left[\left(\overline{N \otimes_{\mathcal{O} H} M^{\top}}\right)(\Delta P)\right]+\left[\left(\overline{N \otimes_{\mathcal{O O H}} N^{\top}}\right)(\Delta P)\right] \\
& =\left(\overline{\dot{\gamma}_{H}^{\gamma}}\right)(\Delta P)=\overline{[\mathcal{O G}]}(\Delta P)=\left[k C_{G}(P) \operatorname{br}_{P}(e)\right] \\
& =\nu\left(\left[\mathcal{O}\left[C_{G}(P)\right] \operatorname{br}_{P}(e)\right]\right)
\end{aligned}
$$

in $T\left(k\left[C_{G}(P)\right], k\left[C_{G}(P)\right]\right)$, and therefore

$$
\gamma(\Delta P)_{C_{H}(P)}^{\cdot} \gamma(\Delta P)^{\swarrow}=\left[\mathcal{O} C_{G}(P) \operatorname{br}_{P}(e)\right]
$$

in $T\left(\mathcal{O}\left[C_{G}(P)\right], \mathcal{O}\left[C_{G}(P)\right]\right)$. Similarly we have

$$
\gamma(\Delta P)^{\sim}{ }_{C_{G}(P)} \gamma(\Delta P)=\left[\mathcal{O} C_{H}(P) \operatorname{br}_{P}(f)\right]
$$

in $T\left(\mathcal{O}\left[C_{H}(P)\right], \mathcal{O}\left[C_{H}(P)\right]\right)$. Applying $\kappa_{C_{G}(P) \times C_{G}(P)}$ to (3.8.a) and $\kappa_{C_{H}(P) \times C_{H}(P)}$ to $3.8 . \mathrm{b}$ we obtain

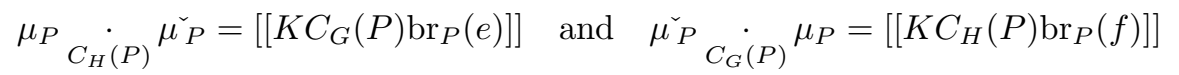

in $R\left(K\left[C_{G}(P)\right], K\left[C_{G}(P)\right]\right)$ and $R\left(K\left[C_{H}(P)\right], K\left[C_{H}(P)\right]\right)$, respectively. Since $M(\Delta P)$ and $N(\Delta P)$ are $\Delta D$-projective, they are projective as left $\mathcal{O}\left[C_{G}(P)\right]$ modules and as right $\mathcal{O}\left[C_{H}(P)\right]$-modules. Therefore, by [Br90, Proposition 1.2], $\mu_{P}$ is perfect, and part (b) is proved.

(c) This follows from [Br90, Theorem 1.5(2)].

(d) The first part follows in the same way as condition (ii) in the proof of Lemma 1.10. The second statement follows from ${ }^{(g, h)} \gamma(\Delta P)=\gamma\left({ }^{(g, h)} \Delta P\right)=$ $\gamma(\Delta Q)$. The third follows from the second and from

$$
{ }^{g}\left(\varepsilon \gamma(\Delta P) \varphi_{C_{H}(P)}^{\cdot} \alpha\right)=g_{\varepsilon}^{(g, h)} \gamma(\Delta P)^{h} \varphi_{C_{H}(Q)}{ }^{h} \alpha .
$$

(e) Condition (i) of Definition 1.9(b) follows from the third statement in part (d) after summing over all $\varepsilon$ and noting that ${ }^{g}\left(\operatorname{br}_{P}(e)\right)=\operatorname{br}_{Q}(e),{ }^{h}\left(\operatorname{br}_{P}(f)\right)=\operatorname{br}_{Q}(f)$, and $\operatorname{br}_{Q}(e) \gamma(\Delta Q) \operatorname{br}_{Q}(f)=\gamma(\Delta Q)$, where $Q={ }^{g} P={ }^{h} P$. 
To prove the commutativity of the diagram in condition (ii) of Definition 1.9(b) we consider the diagram

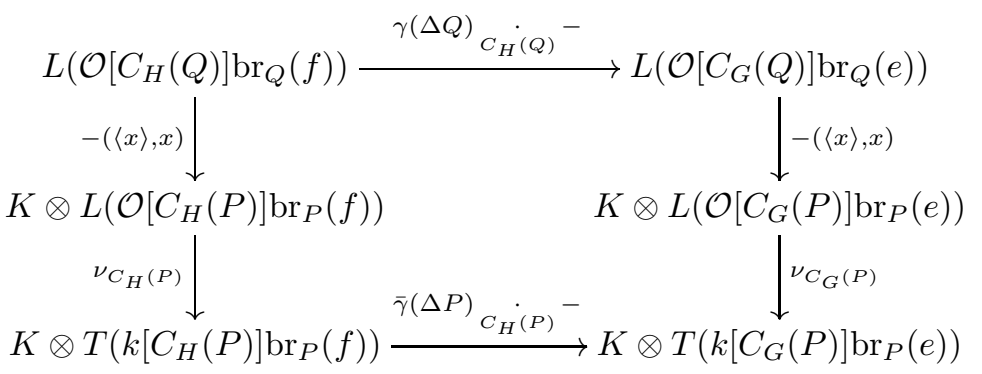

First note that tensoring the trivial source bimodule $\gamma(\Delta Q)$ with a left linear source $\mathcal{O C}_{H}(Q)$-module again results in a linear source module, since $\gamma(\Delta Q)$ is $\Delta D$-projective (cf. Bo01, Proposition 2.3(a)]). Thus, the top horizontal map is well-defined. Next note that the diagram commutes by Corollary [3.6(b) applied to $Y=\gamma(\Delta Q)$ and by using $\bar{\gamma}(\Delta P)=\overline{\gamma(\Delta Q)}(\Delta\langle x\rangle)$ (see Subsection 3.7(a)). The natural maps $\kappa$ and $\eta$ form a morphism between the above square and the square in condition (ii) of Definition [1.9(b) by Theorem 2.4. The required commutativity now follows from the surjectivity of the map $\kappa: L\left(\mathcal{O}\left[C_{H}(Q)\right] \operatorname{br}_{Q}(f)\right) \rightarrow$ $R\left(K\left[C_{H}(Q)\right] \operatorname{br}_{Q}(f)\right)$.

(f) This follows immediately from part (e) and Lemma 1.10(iii) and (iv).

(g) By part (e) and Lemma1.10(i), the conditions in (ii) and (iii) are equivalent. Moreover, (i) implies (ii) by applying the map $\kappa$. Next we show that (ii) implies (i). By the argument in Subsection 3.7(b), it suffices to show that the generalized Brauer character of $(\overline{\varepsilon \gamma(\Delta P) \varphi})(\Delta Q)$ vanishes for all $Q \leqslant D$. But, by Lemma 2.2 and by Subsection 3.7(a), we have

$$
(\overline{\varepsilon \gamma(\Delta P) \varphi})(\Delta Q)=\operatorname{br}_{P Q}(\varepsilon) \bar{\gamma}(\Delta(P Q)) \operatorname{br}_{P Q}(\varphi)
$$

and the Brauer character of the above element is equal to $\operatorname{br}_{P Q}(\varepsilon) \lambda_{P Q} \operatorname{br}_{P Q}(\varphi)$. So assume that the last element is not equal to zero. Then there exist primitive idempotents $\varepsilon^{\prime}$ of $Z\left(\mathcal{O}\left[C_{G}(P Q)\right]\right)$ and $\varphi^{\prime}$ of $Z\left(\mathcal{O}\left[C_{H}(P Q)\right]\right)$ such that $\varepsilon^{\prime} \operatorname{Br}_{P Q}(\varepsilon)=$ $\varepsilon^{\prime}, \varphi^{\prime} \operatorname{Br}_{P Q}(\varphi)=\varphi^{\prime}$ and $\varepsilon^{\prime} \lambda_{P Q} \varphi^{\prime} \neq 0$. Then we have $\zeta\left(P Q, \varphi^{\prime}\right)=\left(P Q, \varepsilon^{\prime}\right),(P, \varepsilon) \leqslant$ $\left(P Q, \varepsilon^{\prime}\right)$, and $(P, \varphi) \leqslant\left(P Q, \varphi^{\prime}\right)$. If we set $(P, \tilde{\varepsilon}):=\zeta(P, \varphi)$, then, by part (f), we also have $(P, \tilde{\varepsilon}) \leqslant\left(P Q, \varepsilon^{\prime}\right)$. But, since also $(P, \varepsilon) \leqslant\left(P Q, \varepsilon^{\prime}\right)$ we obtain $\tilde{\varepsilon}=\varepsilon$ and $\varepsilon \mu_{P} \varphi \neq 0$, a contradiction. This completes the proof of part (g).

(h) By [NT89, Theorem V.5.21] we know that $D$ is a defect group of $\mathcal{O}\left[C_{G}(P)\right] \varepsilon$ and of $\mathcal{O}\left[C_{H}(P)\right] \varphi$, and by part (a) we know that $\varepsilon \gamma(\Delta P) \varphi$ is $\Delta D$-projective. Moreover, if we write $\operatorname{Br}_{P}(e)=\varepsilon_{1}+\cdots+\varepsilon_{n}$ and $\operatorname{Br}_{P}(f)=\varphi_{1}+\cdots+\varphi_{n}$ with $\varepsilon_{1}=\varepsilon, \varphi_{1}=\varphi$, and $\zeta\left(P, \varphi_{i}\right)=\left(P, \varepsilon_{i}\right)$ for $i=1, \ldots, n$, then

$$
\gamma(\Delta P)=\sum_{i=1}^{n} \varepsilon_{i} \gamma(\Delta P) \varphi_{i} \quad \text { and } \quad \gamma(\Delta P)^{`}=\gamma^{`}(\Delta P)=\sum_{i=1}^{n} \varphi_{i} \gamma^{\curlyvee}(\Delta P) \varepsilon_{i},
$$

by part (c). Substituting this in equation (3.8.a) and multiplying both sides with $\varepsilon$ yields

$$
(\varepsilon \gamma(\Delta P) \varphi)_{C_{H}(P)}^{\cdot}(\varepsilon \gamma(\Delta P) \varphi)^{\swarrow}=\left[\mathcal{O} C_{G}(P) \varepsilon\right]
$$


in $T\left(\mathcal{O}\left[C_{G}(P)\right], \mathcal{O}\left[C_{G}(P)\right]\right)$. Similarly, we obtain

$$
(\varepsilon \gamma(\Delta P) \varphi)_{C_{G}(P)}^{\sim}(\varepsilon \gamma(\Delta P) \varphi)=\left[\mathcal{O} C_{H}(P) \varphi\right]
$$

in $T\left(\mathcal{O}\left[C_{H}(P)\right], \mathcal{O}\left[C_{H}(P)\right]\right)$ from equation (3.8.b). Thus, $\varepsilon \gamma(\Delta P) \varphi$ is a $p$-permutation equivalence between $\mathcal{O}\left[C_{G}(P)\right] \varepsilon$ and $\mathcal{O}\left[C_{H}(P)\right] \varphi$, and the theorem is proved.

\section{REFERENCES}

[AB79] J. L. Alperin, M. BrouÉ: Local methods in block theory. Ann. of Math. 110 (1979), 143-157. MR541333 (80f:20010)

[Be84] D. Benson: Modular representation theory: New trends and methods. Springer Lecture Notes 1081, Springer-Verlag 1984. MR765858 (86g:20013)

[Bo98] R. Boltue: Linear source modules and trivial source modules. Proc. Sympos. Pure Math. 63 (1998), 7-30. MR.1603127 (99d:20016)

[Bo01] R. Boltue: Chain complexes for Alperin's weight conjecture and Dade's ordinary conjecture in the abelian defect group case. To appear in J. Group Theory. Available at http://math.ucsc.edu/ boltje/publications.html.

[BK00] R. Boltje, B. Külshammer: A generalized Brauer construction and linear source modules. Trans. Amer. Math. Soc. 352 (2000), 3411-3428. MR1694281 (2000j:20017)

[BK02] R. Boltje, B. Külshammer: Monomial resolutions of trivial source modules. J. Algebra 248 (2002), 146-201. MR1879012 (2002j:20018)

[Br85] M. BrouÉ: On Scott modules and $p$-permutation modules: An approach through the Brauer morphism. Proc. Amer. Math. Soc. 93 (1985), 401-408. MR773988 (86d:20010)

[Br90] M. BrouÉ: Isométries parfaites, types de blocs, catégories dérivées. Astérisque 181-182 (1990), 61-92. MR1051243(91i:20006)

[Ha99] M. HARris: Splendid derived equivalences for blocks of finite groups. J. London Math. Soc. 60 (1999), 71-82. MR.1721816 (2000k:20014)

[NT89] H. Nagao, Y. Tsushima: Representations of finite groups. Academic Press, San Diego 1989. MR.998775 (90h:20008)

[Ri96] J. RICKARD: Splendid equivalences: Derived categories and permutation modules. Proc. London Math. Soc. 72 (1996), 331-358. MR1367082 (97b:20011)

[Th95] J. ThÉvenAz: $G$-algebras and modular representation theory. Oxford University Press 1995. MR 1365077 (96j:20017)

Department of Mathematics, University of California, Santa Cruz, California 95064

E-mail address: boltje@ucsc.edu

Department of Mathematics, University of California, Santa Cruz, California 95064 E-mail address: btxu@math.ucsc.edu

Current address: Department of Mathematics, Eastern Kentucky University, 521 Lancaster Avenue, Wallace 313, Richmond, Kentucky 40475

E-mail address: bangteng.xu@eku.edu 